Please do not remove this page

RMIT

UNIVERSITY

\title{
D-Log: A WiFi Log-based differential scheme for enhanced indoor localization with single RSSI source and infrequent sampling rate
}

Ren, Yongli; Salim, Flora; Tomko, Martin; Bai, Yuntian Brian; Chan, Jeffrey; Qin, Kai; Sanderson, Mark https://researchrepository.rmit.edu.au/esploro/outputs/9921860126101341/filesAndLinks?institution=61RMIT_INST\&index=null

Ren, Y., Salim, F., Tomko, M., Bai, Y. B., Chan, J., Qin, K., \& Sanderson, M. (2017). D-Log: A WiFi Log-based differential scheme for enhanced indoor localization with single RSSI source and infrequent sampling rate. Pervasive and Mobile Computing, 37, 94-114. https://doi.org/10.1016/j.pmcj.2016.09.018 Document Version: Submitted Version

Published Version: https://doi.org/10.1016/j.pmcj.2016.09.018

Repository homepage: https://researchrepository.rmit.edu.au (C) 2016 Elsevier B.V. All rights reserved.

Downloaded On 2023/04/26 18:03:31 +1000 
Thank you for downloading this document from the RMIT Research Repository.

The RMIT Research Repository is an open access database showcasing the research outputs of RMIT University researchers.

RMIT Research Repository: http://researchbank.rmit.edu.aul

\section{Citation:}

Ren, Y, Salim, F, Tomko, M, Bai, Y, Chan, J, Qin, K and Sanderson, M 2017, 'D-Log: A WiFi log-based differential scheme for enhanced indoor localization with single RSSI source and infrequent sampling rate', Pervasive and Mobile Computing, vol. 37, pp. 94-114.

See this record in the RMIT Research Repository at:

https://researchbank.rmit.edu.au/view/rmit:39132

Version: Submitted Version

Copyright Statement:

(C) 2016 ACM.

Link to Published Version:

http://dx.doi.org/10.1145/3015022.3015031 


\title{
D-Log: A WiFi Log-based Differential Scheme for Enhanced Indoor Localization with Single RSSI Source and Infrequent Sampling Rate
}

\author{
Yongli Ren ${ }^{\mathrm{a}}$, Flora Dilys Salim ${ }^{\mathrm{a}}$, Martin Tomko ${ }^{\mathrm{b}}$, Yuntian Brian Bai ${ }^{\mathrm{c}}$, Jeffrey Chan ${ }^{\mathrm{a}}$, Kyle Kai Qin ${ }^{\mathrm{a}}$, Mark \\ Sanderson ${ }^{\mathrm{a}}$ \\ ${ }^{a}$ School of Science, Computer Science and Information Technology, RMIT University, Victoria 3000, Australia \\ ${ }^{b}$ Department of Infrastructure Engineering, The University of Melbourne, Victoria 3010, Australia \\ ${ }^{c}$ School of Math and Geospatial Science, RMIT University, Victoria 3000, Australia
}

\begin{abstract}
Currently, large amounts of Wi-Fi access logs are collected in diverse indoor environments, but cannot be widely used for fine-grained spatio-temporal analysis due to coarse positioning. We present a Log-based Differential (D-Log) scheme for post-hoc localization based on differentiated location estimates obtained from large-scale Access Point (AP) logs of WiFi connectivity traces, which can be used for data analysis and knowledge discovery of visitor behaviours. Specifically, the location estimates are calculated by utilizing a combination of Received Signal Strength Indicator (RSSI) records from two neighbouring APs. D-Log exploits real-world industry WiFi logs where RSSI data sampled at low rates from single AP sources are recorded in each connectivity trace. The approach is independent of device and network infrastructure type. D-Log is evaluated using WiFi logs collected from controlled environment as well as real-world uncontrolled public indoor spaces, which includes discrete single-AP RSSI traces of around 100,000 mobile devices over a one-year period. The experiment results indicate that, despite of the challenges with the infrequent sampling rate and the limitations of the data that only records RSSI from single AP sources in each instance, DLog performs comparatively well to the state-of-the-art RSSI-based localization methods and presents a viable alternative for many application areas where high-accuracy positioning infrastructure may not be cost effective or where positioning applications are considered on legacy information infrastructure.
\end{abstract}

Keywords: RSSI, WiFi log, localization

\section{Introduction}

The use of a RSSI from multiple WiFi APs to estimate the position of mobile devices in a wireless networked environment is a well established procedure. Three main approaches are commonly used when RSSI traces are available: trilateration, scene analysis (WiFi fingerprinting), and proximity-based localization. Most of these methods aim to generate an accurate estimate of a mobile device's position in the networked environment. Furthermore, these approaches often demand either that the WiFi networks are configured for high sampling rates and continuous monitoring from multiple access points, or require users to install an app on their device for data collection. This leads to implementation barriers such as high setup, engineering, and calibration cost and the requirements for user participation. Hence, there is a need for approaches applicable to low sampling rates and single access point monitoring. Another source of data that has thus far been barely examined for enhancing localization: large volumes of WiFi AP logs of non-continuous WiFi connectivity traces that are normally stored in an external system, representing timestamped connections between a device and a single Access Point, along with the associated RSSI. With such data, a research

Email addresses: yongli.ren@rmit.edu.au (Yongli Ren), flora.salim@rmit.edu.au (Flora Dilys Salim), tomkom@unimelb.edu.au (Martin Tomko), yuntianbrian.bai@rmit.edu.au (Yuntian Brian Bai), jeffrey.chan@rmit.edu.au (Jeffrey Chan), kyleqin2008@gmail.com (Kyle Kai Qin), mark.sanderson@rmit.edu.au (Mark Sanderson) 


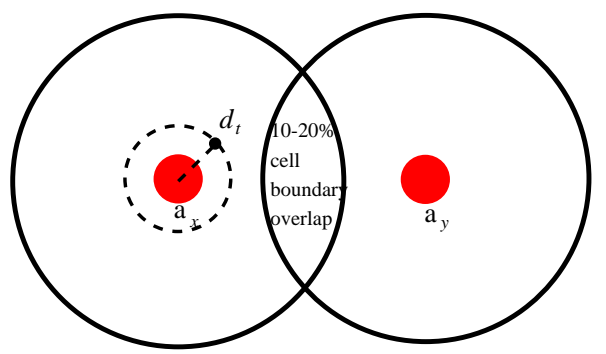

(a)

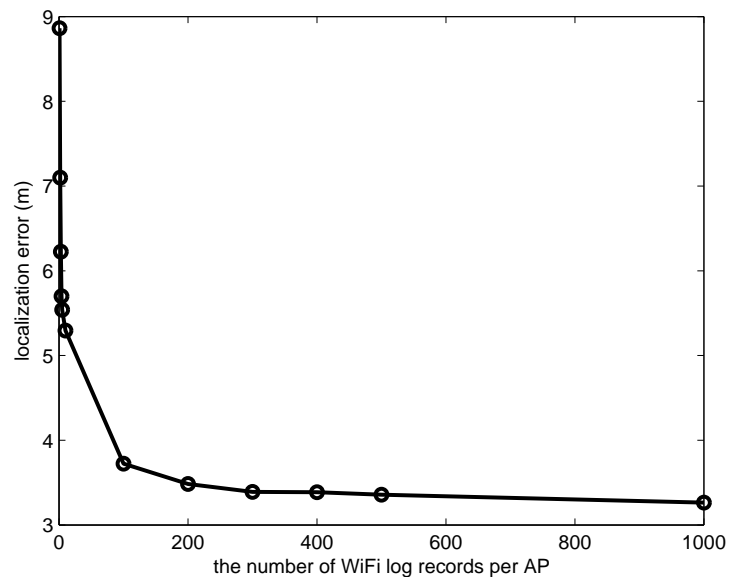

(b)

Figure 1: (a) Coverage areas of two adjacent APs and the cell boundary overlap. The overlapping area is $10-20 \%$ of a cell's area. (b) An experimental illustration of the dependence of the accuracy of the method on the number of available RSSI observations during handover.

question emerges:

How to perform accurate indoor localization using large-scale logs of discrete single-AP RSSI traces with low sampling rate?

This problem opens a new direction for localization research. Specifically, we describe a robust WiFi logbased localization scheme which is:

1. non-intrusive: it expects nothing from the client mobile device, e.g. there is no need to install an app, turning-on of sensors other than WiFi;

2. generic: it is simple to deploy and applicable in any WiFi installation, which has an overlap between the coverage areas of adjacent APs and is capable of recording RSSI values when handovers occur between them. Additionally, the knowledge of the relative transmitter output power of the APs should be known by the operator;

3. light-weight: it uses algorithms that are simple to implement and maintain and do not overload existing computational infrastructures;

4. effective: as long as a mobile device connects to the WiFi network, the localization technique can be applied; and

5. accurate: the scheme delivers accuracy that is comparable to scene analysis, and exceeds the classical path loss model $[1,2]$, as demonstrated in the evaluation.

The D-Log positioning method is an enhancement over existing methods that roughly localize a device anywhere in the service area of an AP, by providing an estimate of the distance between the mobile device and the connected AP. This allows to further restrict the space in which the device is found. D-Log focuses on static localization, not the continuous tracking of people's movement.

D-Log works by improving distance estimations from discrete single-AP RSSI traces of a mobile device. Specifically, D-Log applies the WiFi path loss model in combination with knowledge of the distance of neighbouring APs in a WiFi network and the probability distribution of each logged RSSI record to better estimate this distance. The key point is to utilize a combination of RSSI records from two neighbouring APs where handovers occur: a location that is known with some certainty [3, 4]. This information can be used to reduce errors introduced from the path loss model. 
D-Log computes an enhanced distance estimate of a mobile device within the region served by a given AP for each individual logged RSSI record. D-Log treats these estimates as independent instances drawn from the same distribution. Applying probability theory, the average of these measurements allows estimation, with greater accuracy, of the distance between the AP and the mobile device. The theoretical analysis is provided to show D-Log's performance in terms of localization accuracy (Section 3.5).

Consider two neighbouring APs $a_{x}$ and $a_{y}$ and the mobile device at the distance $d_{t}$, served by $\operatorname{AP} a_{x}$, as shown in Fig. 1a. D-Log calculates one estimate of $d_{t}$ by using each logged RSSI value when a handover occurred. As there are a large number of WiFi log records for numerous devices, D-Log obtains a large number of estimates of $d_{t}$ at handover, and uses them to determine the average estimate as the distance to the handover location $d_{t}$. This allows us to establish the empirical signal strength decay progression around an AP, along which any non-handover locations can be interpolated for any observation of RSSI. Thus, we use the knowledge of the handover to calibrate the signal strength decay function based on a path loss model for each AP. Fig. 1b illustrates the dependence of the accuracy of the D-Log method on the number of RSSI observations during handover, based on the experiments discussed later. As the number of logged RSSI records increases, the average error of the position estimate decreases (Fig. 1b), converging towards a limit value little above $3.0 \mathrm{~m}$, achieved at around 300 observations.

Once a sufficient amount of WiFi AP logs has been recorded, they can be used to train the D-Log algorithm. D-Log can then be used in (near) real-time, similar to other existing RSSI-based localization methods. The D-Log scheme is, however, primarily meant to be deployed to improve the location estimate in mobile device access records collected in a WiFi system in a post-processing step. Note that such logs are collected at infrequent sampling rates from a single RSSI source to which the device is connected to. Most existing RSSI-based methods are infeasible in such scenarios. Such enhancement of location estimation is important for the improvement of indoor context estimation supporting a range of applications exploiting indoor behaviour information mining and recommender systems $[5,6]$, in environments with free and publicly available WiFi networks. Potential application areas include retail and advertising (e.g. shopping malls, airports), leisure and tourism (e.g. attractions, entertainment areas), rich media consumption (e.g. smart displays), teaching and learning support (e.g. in universities), and operational logistics (e.g. in airports, transport hubs). Once accurate post-hoc localization of users within indoor spaces is possible, large-scale Web activity and connectivity logs from the WiFi systems will enable extensive indoor information behaviour mining and long-term prediction of user behaviours $[7,8]$.

The remainder of the paper is organized as follows. Section 2 presents the related work. The D-Log scheme is detailed in Section 3, where a theoretical analysis is provided to show the performance benefit of D-Log. Section 4 presents the data that we experiment with. Section 5 includes the evaluation of the proposed method, and Section 6 concludes the paper and discusses possible future research.

\section{Related Work}

\subsection{Indoor localization techniques}

Existing research on indoor localization can be categorized into device-based [9, 10, 11, 12, 13], device-free (passive) localization [14, 15], and infrastructure-based localization[16, 17, 18].

Device-based localization has gained popularity in recent years. This is due to the ability to integrate data from multiple smartphone sensors (e.g. [19]) and thus allow for the combination of dead reckoning [12, 20, 21] and particle filter estimation methods [22]. Although such a rich combination of signals improves indoor localization, this is outside the scope of this paper, which is focused on post-hoc localisation based on (sparse) WiFi AP logs of all the registered WiFi users. For device-based localization, it requires on-device processing, typically via a mobile app, as well as continuous sampling of data. Given the requirement of user participation and uptake with a mobile app, it limits the coverage of indoor monitoring. Full coverage is often considered as a major requirement for indoor monitoring by facility owners and operators.

The most recent, albeit less common technique is device-free (passive) localization [14, 15]. Mobile device-free localization does not require a device attached or carried by indoor visitors. But such methods require high and continuous sampling rates and substantial post-processing efforts. They operate well 
only in controlled environments, and multi-user tracking capability is often limited to small numbers of simultaneously tracked objects. The most recent device-free (passive) localization method is capable of tracking three users simultaneously [23]. Given the challenges with multi-user tracking and the need for highly densed monitoring points and RSSI sampling, this is not applicable for tracking users in large-scale public indoor spaces.

Many infrastructure based techniques utilise trilateration, which requires RSSI from multiple nearby APs. However, these techniques are expensive to implement, since the WiFi networks have to be deployed with a data logging configuration allowing multiple access points to be monitored across each device connection for passive localization. This is typically not the case with most indoor environments currently operating WiFi networks. As such, the logs acquired cannot be mined for accurate indoor spatial behaviour estimation.

Some research employs fusion of techniques. In [21], in-device recorded RSSI from a single access point is used, however, the technique relies on dead reckoning to provide a perceived triangulation on the device. Khan et. al. improved the coverage of localization through active participation of users [24]. Other localization techniques employ the use of ZigBee networks (e.g. [25, 26]), RFID tags [27], or propagation model and autonomous crowdsourcing [28].

\subsection{RSSI use in indoor localization}

With regard to the use of RSSI from WiFi access points in localizing devices of a WiFi network, traditionally, there are three main methods that are widely employed: trilateration, scene analysis, and proximity analysis $[29,30]$.

First is trilateration, which estimates the position of a device by calculating its distance from multiple reference points [30]. When RSSI traces from multiple access points are available, the use of this path loss based method is a more accurate approach to localize a device, rather than using Time-of-Arrival or Time-Difference-of-Arrival calculation [30] to approximate a device location, as the latter two methods require a clear Line-Of-Sight (LOS) between the transmitter and the receiver [30]. An example of the use of trilateration is in [17], where WiFi RSSI traces from multiple reference (access) points were recorded in order to monitor around 18,000 devices in a hospital. They used WiFi signals measured on mobile devices to first localize users in the building, extracted the spatial and temporal features from the traces, analyzed the flow of people from entrance to exit, and classified their behaviours based on the user roles [17]. However, in our study, RSSI from multiple reference points are not available, hence, trilateration is not applicable.

The second established RSSI-based localization approach is Radio Frequency (RF) based scene analysis, a method to use prior-collected features, or fingerprints, of a scene to determine the location [29]. The most widely used scene analysis method is RSSI-based fingerprinting [30]. Swangmuang and Krishnamurthy presented an analytical model to predict the performance of fingerprinting-based indoor localization systems by applying proximity graphs [31]. A WiFi RSSI fingerprint for each location is used to match the monitored (indoor) environment for accurate localization of the device [32]. In some cases, fingerprinting at the actual site is not feasible, e.g., in a very large shopping mall or airport. Since fingerprinting requires a large amount of time and resources and costly system calibration in the beginning [32], the real-world use of this approach was difficult. For example, in a highly dynamic environment, where layouts and objects often change, $\mathrm{RF}$ fingerprints could easily change due to alterations of the indoor environment, hence requires frequent fingerprinting [12]. [33] used knowledge about the geometry of the environment and made assumptions about continuous indoor movement tracking to address this problem, while [34] collected user feedback to improve the fingerprinting process. Want et. al. proposed a combination of subarea fingerprinting and gradient descent search to improve localization by probabilistic fitting [35], but this fingerprinting approach requires high frequency sampling.

The third approach is proximity-based localization, which uses RSSI captured on users' devices to compute approximate sets of devices that are located in proximity to each other to localize the position of a device relative to another device [29]. This method does not apply in our study since we do not use apps or device-based approach to localize a user.

In this paper, we propose the D-Log scheme as a new reference scheme for post-hoc localization, which aims to be easy to implement and maintain, is independent of devices and network infrastructure, and is effective and reasonably accurate. In Table 1, we compare D-Log with existing schemes, including trilateration, 
Table 1: Comparison of indoor localization schemes.

\begin{tabular}{|c|c|c|c|c|c|c|c|}
\hline Schemes & Signal & Cost & $\begin{array}{l}\text { Client } \\
\text { Sensors } \\
\text { /Apps }\end{array}$ & $\begin{array}{l}\text { AP } \\
\text { Place- } \\
\text { ments }\end{array}$ & $\begin{array}{l}\text { RSSI Source } \\
\text { (No. of } \\
\text { APs) }\end{array}$ & Sampling Rate & Comments \\
\hline Trilateration & RSSI & Med & $\mathrm{No}$ & Normal & At least 3 & Low (continuous) & Infrastructure-based \\
\hline Scene analysis & $\begin{array}{l}\text { RSSI\& } \\
\text { Sensors }\end{array}$ & High & Yes & Normal & Multiple & High (continuous) & Device-based \\
\hline Proximity analysis & RSSI & High & Yes & Dense & Multiple & High (continuous) & Device-based \\
\hline Device free & RSSI & High & Yes & Dense & Multiple & High (continuous) & Device-free/passive \\
\hline D-Log & RSSI & Low & No & Normal & Single & Low (discrete) & Log-based \\
\hline
\end{tabular}

scene analysis, proximity analysis and device free approaches in terms of their deployment characteristics. The D-Log scheme is low cost, because it only requires infrequent RSSI sampling from single RSSI source, rather than continuous RSSI sampling from multiple RSSI sources like others (e.g. scene analysis).

\section{Log-Based Differential Scheme}

In this section, we formulate the targeted research question and present two D-Log algorithms to estimate the distance of the mobile device to the AP. Furthermore, the complexities of the D-Log algorithms are analysed, and a theoretical analysis is provided to show the performance benefit of the entire proposed D-Log scheme.

\subsection{Problem Formulation}

In this paper, the research question is the estimation of a mobile device location within the coverage area of several WiFi APs based on logs of discrete RSSI traces from single APs. We assume that the WiFi log includes discrete RSSI measurements relating to a single AP connection at any one time, in contrast to the trilateration and scene analysis methods requiring multiple parallel RSSI observations. Single RSSI records are recorded in most real-world Wi-Fi system data logs, where non-serving APs and their RSSI are not recorded. Although these single-AP RSSI traces are normally discrete and sampled at low frequency, the quantity of records obtained from different devices for each WiFi AP is large. For example, the realworld WiFi log we examined (as detailed in Section 4), was collected with a 5min sampling rate for each registered mobile device; logging only the RSSI values for currently connected APs. This resulted in 480,924 connections distributed amongst $35 \mathrm{APs}$, with in average around 13,000 records per AP. This large volume of available records for each AP creates an opportunity to accurately estimate the distance of a mobile device from an AP given its RSSI value.

There are several techniques to calculate $d_{t}$ given an RSSI value $r_{t}$ for a mobile device when associating with an AP. The path loss model $[1,2]$ enables to determine the device distance based on the full set of inputs:

$$
\hat{d}_{t}=10^{\left(\frac{T X_{p w r}-r_{t}-L_{t x}-L_{r x}+G_{t x}+G_{r x}-P L-s}{10 e}\right)}
$$

where $\hat{d}_{t}$ denotes the estimated distance between the transmitter and the receiver (the client mobile device) in meters; $T X_{p w r}$ is the transmitter output power in $\mathrm{dB} ; r_{t}$ is the detected RSSI in $\mathrm{dB} ; L_{t x}$ is the sum of all transmitter-side cable and connector losses in $\mathrm{dB} ; L_{r x}$ is the sum of all receiver-side cable and connector losses in dB; $G_{t x}$ is the transmitter-side antenna gain in $\mathrm{dBi} ; G_{r x}$ is the receiver-side antenna gain in $\mathrm{dBi}$; $P L$ is the reference path loss in $\mathrm{dB}$ for the desired frequency when the receiver-to-transmitter distance is one meter; $s$ is the standard deviation associated with the degree of shadow fading present in the environment; $e$ denotes the path loss exponent for the environment. Note, although Eq. 1 takes a range of factors into consideration, the estimation of $\hat{d}_{t}$ is not accurate, as the RSSI values $r_{t}$ at location $p_{x}$ vary and can be affected by a large number of external factors, e.g. the people movement through the space, the layout of the walls and the materials used in the environment. 


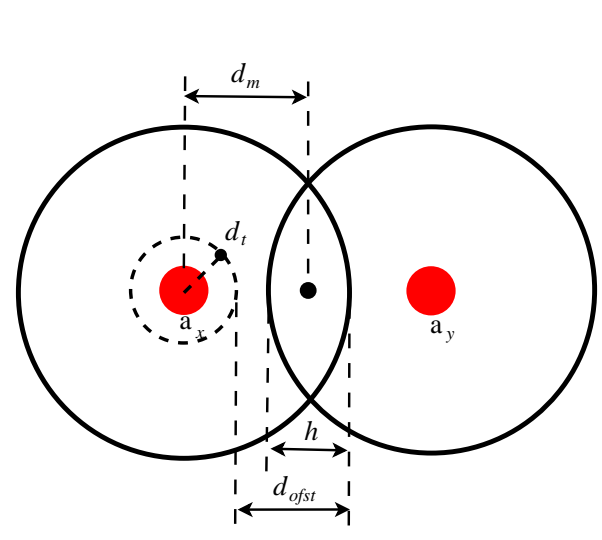

(a) Theoretically circular shaped coverage

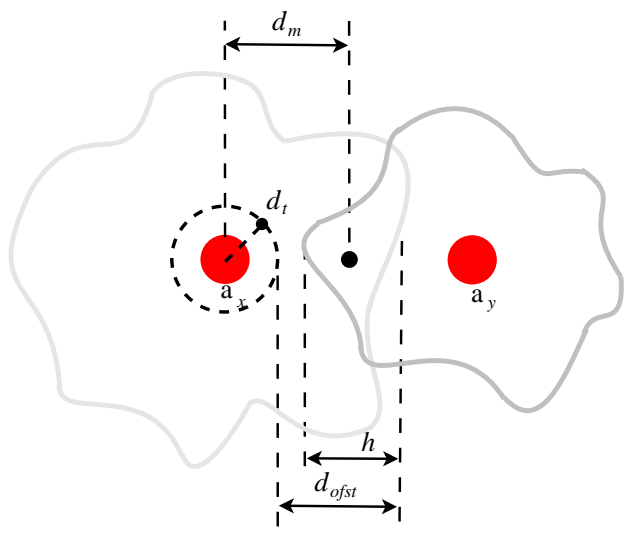

(b) Practically irregular-shaped coverage

Figure 2: Illustration of $d_{m}, h$ and $d_{o f s t}$ in D-Log algorithm with both theoretically circular shaped and practically irregular shaped coverage of several Wi-Fi APs. Here, the irregular shaped Wi-Fi AP coverage is obtained by following the study of wireless performance and coverage from Cisco Meraki [36].

Let us consider a general case: given two sets of sample RSSI values $\mathcal{R}_{x}$ and $\mathcal{R}_{y}$, collected when the handover between two adjacent access points $a_{x}$ and $a_{y}$ happens, we denote $r_{x}^{i} \in \mathcal{R}_{x}$ a sample RSSI value observed when a mobile device is disassociating with $a_{x}$ and then immediately associating with $a_{x}$ 's topological adjacent AP $a_{y}$; similarly, each $r_{y}^{i} \in \mathcal{R}_{y}$ denotes a sample RSSI value observed when a device is disassociating with $a_{y}$ and then immediately associating with $a_{x}$. As there is only one observed RSSI value to the connected AP for the mobile device at any time, then other methods that rely on concurrent RSSI measurements from multiple APs are not applicable (e.g. trilateration and scene analysis). To address this problem, we propose the D-Log scheme to estimate $d_{t}$ from the RSSI records $r_{x}^{i} \in \mathcal{R}_{x}$, not from $r_{t}$ directly. Specifically, D-Log computes three other distances to interpolate $d_{t}: 1$ ) the distance $d_{m}$ of mid-point of the overlapping coverage areas between $a_{x}$ and $\left.a_{y} ; 2\right)$ the size, $h$ of the handover area between $a_{x}$ and $\left.a_{y} ; 3\right)$ the offset $d_{\text {ofst }}$ between the mobile device and the handover boundary of $a_{x}$. As the two RSSI observations at handover have a number of inputs identical (assuming the transmitting power of the APs is either known or their proportions are known), this differential scheme allows to reduce the number of degrees of freedom influencing the distance determination. This indirect estimation enables D-Log to obtain a large number of distinct estimates for $d_{m}, h$ and $d_{o f s t}$, respectively, because there are a large number of $r_{x}^{i} \in \mathcal{R}_{x}$ in the $\log$. As $r_{x}^{i} \in \mathcal{R}_{x}$ was collected independently in the log, the estimates from them are thus independent to each other. Then, from the aspect of probability theory, these observations can be used to estimate $d_{m}, h$ and $d_{o f s t}$, respectively. Take $d_{m}$ as an example,

$$
\hat{\mu}\left(d_{m}\right)=E\left(d_{m} \mid r_{x}\right)=E\left(\hat{d}_{m}^{i}\right)=\frac{1}{n} \sum_{i=1}^{n} d_{m}^{i},
$$

where $d_{m}^{i}$ is the estimated distance of $d_{m}$ based on a logged RSSI value $r_{x}^{i}$, and $n$ is the number of log records. Moreover, this estimator has large practical application, as large datasets of RSSI logs are common and useful for a number of applications. Thus, the final interpolated $d_{t}$ is accurate, and this will be detailed in the following sections.

\subsection{D-Log Algorithm}

Here, we propose the basic D-Log algorithm to estimate the location of a mobile device within the coverage area of an AP. The D-Log algorithm performs the localization using the following four steps:

- Step 1: Estimation of the distance $d_{m}$ for the mid-point $p_{m}$ of the overlapping coverage areas of two adjacent APs, $a_{x}$ and $a_{y}$. Given a set of the RSSI values $r_{x}^{i} \in \mathcal{R}_{x}$ and $r_{y}^{i} \in \mathcal{R}_{y}$, obtained when the 
handover happens between $a_{x}$ and $a_{y}$, we define that

$$
\hat{d}_{m}=E\left(\hat{d}_{m}^{i}\right)=\frac{1}{n} \sum_{i=1}^{n} \hat{d}_{m}^{i}=\frac{1}{n} \sum_{i=1}^{n} \frac{\hat{d}_{x}^{i}-\hat{d}_{y}^{i}+D}{2},
$$

where $n$ denotes the number of sample RSSI values in $\mathcal{R}_{x}$ and $\mathcal{R}_{y}, D$ is the known distance between $a_{x}$ and $a_{y}$, and $\hat{d}_{x}^{i}$ and $\hat{d}_{y}^{i}$ are the estimate distance from $r_{x}^{i}$ and $r_{y}^{i}$ by using Eq. 1, representing the distance from where the handover occurs to $a_{x}$ and $a_{y}$, respectively.

- Step 2: Estimation of the size of the handover area of two adjacent APs:

$$
\hat{h}=E\left(\hat{h}^{i}\right)=\frac{1}{n} \sum_{i=1}^{n} \hat{h}^{i}=\frac{1}{n} \sum_{i=1}^{n}\left(\hat{d}_{x}^{i}+\hat{d}_{y}^{i}-D\right) .
$$

- Step 3: Estimation of the offset between the mobile device at $p_{t}$ and the handover boundary of the access point $a_{x}$.

$$
\hat{d}_{o f s t}=E\left(\hat{d}_{o f s t}^{i}\right)=\frac{1}{n} \sum_{i=1}^{n} \hat{d}_{o f s t}^{i}=\frac{1}{n} \sum_{i=1}^{n}\left(\hat{d}_{x}^{i}-\dot{\hat{d}}_{t}\right),
$$

where $\dot{\hat{d}}_{t}$ denotes the estimate distance from $p_{t}$ to AP $a_{x}$ by Eq. 1 .

- Step 4: Calculation of the distance of the mobile device at $p_{t}$ within the signal coverage area of $a_{x}$.

$$
\hat{d}_{t}=\hat{d}_{m}+\frac{\hat{h}}{2}-\hat{d}_{o f s t}
$$

Note, Eq. 6 differentiates the estimate of $\hat{d}_{t}$ from each $r_{x}^{i}$ and $r_{y}^{i}$ via Eq. 3, 4, and 5 from Step 1, 2 and 3. Thus, the D-Log algorithm can provide accurate localization of a mobile device within the coverage area of $a_{x}$. Once the distance to the mid point and the interpolation of RSSI values of $a_{x}$ are determined, they can be applied to locate the mobile device at any distance from the serving AP as long as they are within the range. In addition, Fig. 2 shows an illustration of $d_{m}, h$ and $d_{o f s t}$ in D-Log algorithm. Specifically, Fig. 2a shows these parameters when the Wi-Fi AP coverage shape is considered as circles theoretically, while Fig. $2 \mathrm{~b}$ shows them when the coverage shape is irregular in practice.

\subsection{Weighted D-Log Algorithm}

The WiFi logs can be used to determine the distribution of the RSSI values when the handover happen between two adjacent APs $a_{x}$ and $a_{y}$. Fig. 3 shows the distribution of these RSSI values collected in a realworld WiFi infrastructure in a large shopping mall in Australia (detailed in Section 4), and it is observed that they do not follow a uniform distribution. Highly frequent observations of the RSSI (here, around 2000 RSSI observations with $r=-70 \mathrm{~dB}$ ) bear higher impact on the final D-Log estimate than the less frequent ones (e.g. the 400 observations with $r=-90 \mathrm{~dB}$ ). Commercial WiFi networks optimized for coverage often set $-70 d B$ as a threshold value for received signal strength [37]. Following this, we propose a weighted D-Log algorithm by taking the RSSI sample frequency into consideration. Thus, we define the weighted version of the simple expectation location estimator (in Eq. 2) as:

$$
\hat{\mu}\left(d_{m}\right)=E\left(d_{m} \mid r_{x}\right)=E\left(\hat{d}_{m}^{i}\right)=\frac{1}{\sum_{i}^{u} c_{x}^{i}} \sum_{i=1}^{u} c_{x}^{i} d_{m}^{i},
$$

where $c_{x}^{i}$ is the frequency of $r_{x}^{i}, u$ denotes the number of unique $r_{x}^{i}$, and $\sum_{i}^{u} c_{x}^{i}=n$. 


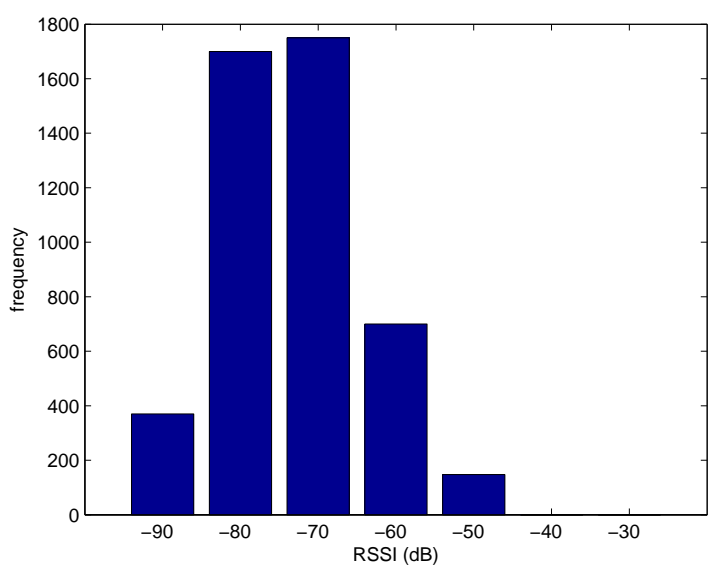

Figure 3: Distribution of RSSI values when handover happen between two adjacent APs in a real-world WiFi log, discussed in Section 4

Therefore, the corresponding weighted versions of $\hat{d}_{m}, \hat{h}, \hat{d}_{o f s t}$ and $\hat{d}_{t}$ are defined as:

$$
\begin{aligned}
\hat{d}_{m}^{\prime} & =E\left(\hat{d}_{m}^{i}\right)=\sum_{i=1}^{u} \frac{w_{x}^{i}\left(\hat{d}_{x}^{i}-\hat{d}_{y}^{i}+D\right)}{2}, \\
\hat{h}^{\prime} & =E\left(\hat{h}^{i}\right)=\sum_{i=1}^{u} w_{x}^{i}\left(\hat{d}_{x}^{i}+\hat{d}_{y}^{i}-D\right), \\
\hat{d}_{o f s t}^{\prime} & =E\left(\hat{d}_{o f s t}^{i}\right)=\sum_{i=1}^{u} w_{x}^{i}\left(\hat{d}_{x}^{i}-\dot{\hat{d}}_{t}\right), \\
\hat{d}_{t}^{\prime} & =\hat{d}_{m}^{\prime}+\frac{\hat{h}^{\prime}}{2}-\hat{d}_{o f s t}^{\prime},
\end{aligned}
$$

where $w_{x}^{i}=\frac{c_{x}^{i}}{\sum c_{x}^{i}}$, and $c_{x}^{i}$ denotes the frequency of sample $r_{x}^{i}$.

\subsection{Complexity Analysis}

One advantage of the proposed D-Log scheme is its low computational complexity. The complexity of the D-Log algorithm is $O(n)$, where $n$ denotes the average number of log records per AP; the complexity of the weighted D-Log algorithm is $O(u)$, where $u$ denotes the number of unique RSSI values per AP. This indicates that D-Log scheme is efficient and only depends on the local log records for neighbouring APs, which enables the processing of large volume of records in parallel. In contrast, the complexity of the other RSSI based localization methods are often much larger than D-Log. For example, the complexity of machine learning based scene analysis (fingerprinting) models, is the same as that of the deployed machine learning methods, e.g, the complexity of SVM-based localization method is $O\left(\max (n a, a) \cdot \min (n a, a)^{2}\right)[38]$, where $n$ is the number of training records, and $a$ is the number of APs.

\subsection{Performance Analysis}

In this section, we provide a theoretical analysis of the performance of the unweighted D-Log algorithm.

The distance from where each $r_{x}^{i}$ is observed to $a_{x}$ can be estimated with Eq. 1, although there is an error $\varepsilon$ caused by systematic and stochastic factors. For access point $a_{x}$, we define the estimation from $r_{x}^{i}$ as

$$
\hat{d}_{x}^{i}=d_{x}^{i}+\varepsilon_{x}^{i}
$$




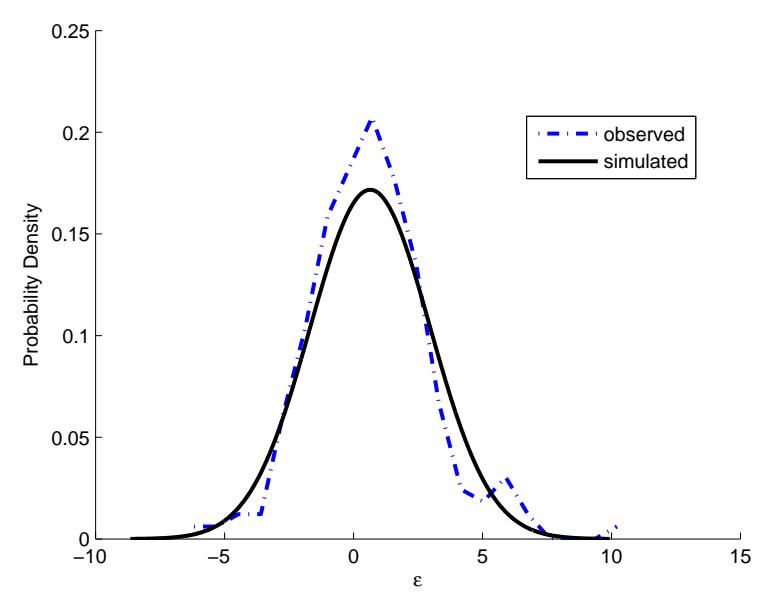

(a) The distribution

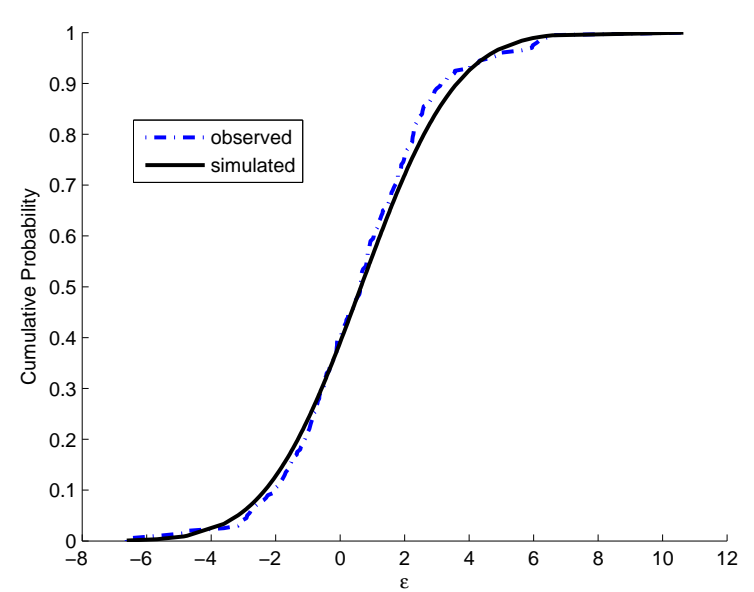

(b) The (ECDF) CDF

Figure 4: The distribution and (ECDF) CDF of $\varepsilon$ and the reference Gaussian distribution

where $\hat{d}_{x}^{i}$ is the distance estimation from $r_{x}^{i}$ with Eq. $1, d_{x}^{i}$ is the real distance, and $\varepsilon_{x}^{i}$ is the error for this estimation. Then, for access point $a_{y}$, we obtain

$$
\hat{d}_{y}^{i}=d_{y}^{i}+\varepsilon_{y}^{i} .
$$

We further assume that the estimation error $\varepsilon$ from each sample RSSI value is independent and identically distributed (i.i.d), and we adopt the Gaussian distribution for theoretical analysis. This is motivated from the experimental results. Specifically, Fig. 4a shows the distribution of $\varepsilon$ in our controlled experiment, which is detailed in Section 4. The dashed blue line depicts the observation empirical distribution of $\varepsilon$ in the experiment, and the solid black line depicts the reference Gaussian distribution with the mean and standard deviation of $\varepsilon$. Fig. $4 \mathrm{~b}$ shows the Empirical distribution function (ECDF) of $\varepsilon$ (the dashed blue line) and the Cumulative Distribution Function(CDF) of the reference Gaussian distribution. It is observed that the reference Gaussian distribution fits the observation distribution of $\varepsilon$ (with $D=0.0558, p$-value $=0.5609$ in Kolmogorov-Smirnov test), and it is thus a suitable model for the following theoretical analysis.

Consequently, the Probability Density Function (PDF) of $\varepsilon$ is:

$$
p(\varepsilon) \sim N\left(\mu_{\varepsilon}, \sigma_{\varepsilon}^{2}\right) .
$$

As stated in Eq. 2, we measure $\hat{d}$ by applying the sample mean as the location estimator, and the distance on each observed RSSI can be considered as an observation. In the first step of D-Log algorithm, for the calculation of $\hat{d}_{m}$, according to Eq. 3 and Eq. 14, we obtain

$$
\hat{d}_{m}=E\left(d_{m}^{i}\right)=\frac{1}{n} \sum_{i=1}^{n} \frac{\hat{d}_{x}^{i}-\hat{d}_{y}^{i}+D}{2}=\frac{1}{2}\left(d_{x}-d_{y}+D\right)+\frac{1}{2 n} \sum_{i=1}^{n}\left(\varepsilon_{x}^{i}-\varepsilon_{y}^{i}\right),
$$

where $d_{x}$ and $d_{y}$ are the real distances of the handover boundary for $a_{x}$ and $a_{y}$, respectively. Similarly,

$$
\hat{h}=E\left(\hat{h}^{i}\right)=\left(d_{x}+d_{y}-D\right)+\frac{1}{n} \sum_{i=1}^{n}\left(\varepsilon_{x}^{i}+\varepsilon_{y}^{i}\right) .
$$

For the estimation of the offset between the mobile device at $p_{t}$ and the handover boundary of the access point $a_{x}$, according to Eq. 5 and Eq. 14, we obtain

$$
\hat{d}_{o f s t}=E\left(\hat{d}_{o f s t}^{i}\right)=\frac{1}{n} \sum_{i=1}^{n}\left(\hat{d}_{x}^{i}-\dot{\hat{d}}_{t}\right)=\left(d_{x}-d_{t}\right)+\frac{1}{n} \sum_{i=1}^{n}\left(\varepsilon_{x}^{i}-\varepsilon_{t}\right),
$$




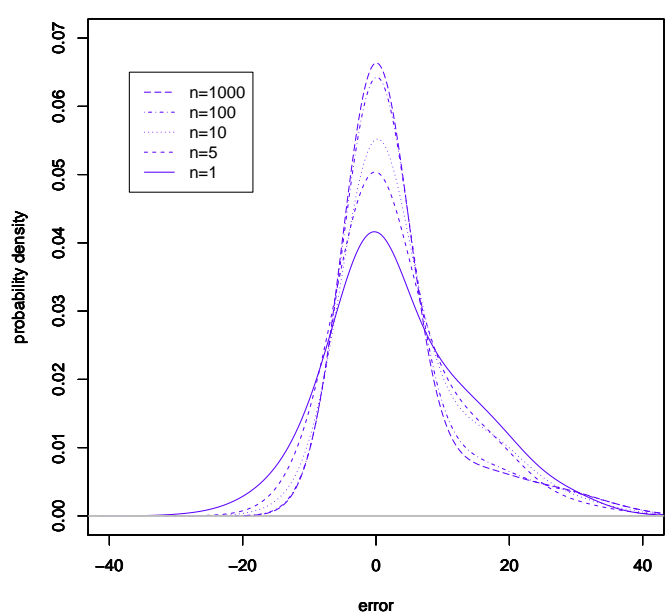

(a) The distribution of $\varepsilon_{\hat{d}_{t}}$ with various $n$

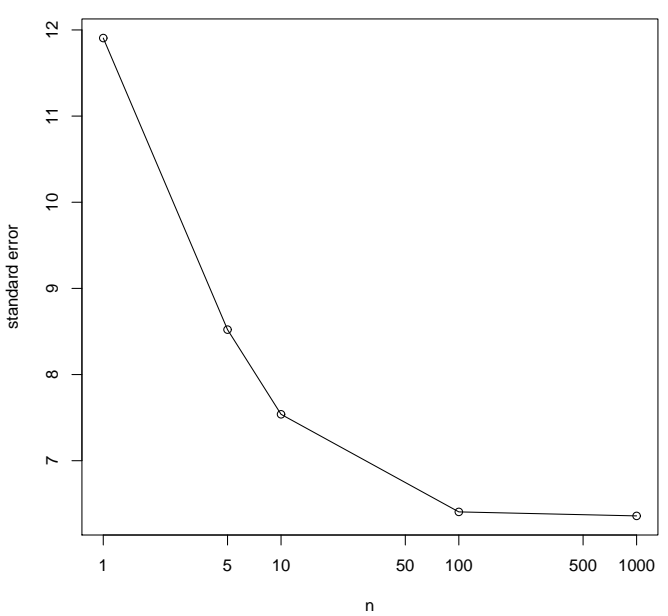

(b) the trend of the standard error $\sigma_{\hat{d}_{t}}$ with various $n$

Figure 5: The impact of $n$ on $\varepsilon_{\hat{d}_{t}}$ and $\sigma_{\hat{d}_{t}}$

where $d_{t}$ is the real distance between the test point $p_{t}$ to $a_{x}$, and $\varepsilon_{t}$ is the error when calculating $\dot{\hat{d}}_{t}$.

Consequently, in the last step of D-Log, according to Eq. 6, Eq. 15, Eq. 16, Eq. 17 and Eq. 14, we obtain:

$$
\hat{d}_{t}=\hat{d}_{m}+\frac{\hat{h}}{2}-\hat{d}_{o f s t}=d_{t}+\frac{1}{2 n} \sum_{i=1}^{n}\left(\varepsilon_{x}^{i}-\varepsilon_{y}^{i}\right)+\frac{1}{n} \sum_{i=1}^{n}\left(\varepsilon_{x}^{i}+\varepsilon_{y}^{i}\right)-\frac{1}{n} \sum_{i=1}^{n}\left(\varepsilon_{x}^{i}-\varepsilon_{t}\right) .
$$

Thus, according to Eq. 18 and Eq. 14, we obtain the $100(1-\alpha) \%$ confidence interval $C I\left(\hat{d}_{t}\right)$ for the estimation of $\hat{d}_{t}$, which has been widely used to indicate the reliability of an estimation [39],

$$
C I\left(\hat{d}_{t}\right)=d_{t} \pm z_{\frac{\alpha}{2}} \sqrt{\frac{5 \sigma_{\varepsilon}^{2}}{n}}
$$

where $z_{\frac{\alpha}{2}}$ is a standard normal variate which exceeded with a probability of $\frac{\alpha}{2}$. Therefore, the standard error of $\hat{d}_{t}$ is:

$$
\sigma_{\hat{d}_{t}}=\sqrt{\frac{5 \sigma_{\varepsilon}^{2}}{n}}
$$

where $n$ denotes the sample size.

Theorem 1. The standard error $\sigma_{\hat{d}_{t}}$ of D-Log scheme is bounded to be no more than $\sqrt{5 \sigma_{\varepsilon}^{2}}$, with equality if and only if $n=1$.

PROOF 1. As the sample size $n \geq 1$, based on Eq. 20, we obtain:

$$
\sigma_{\hat{d}_{t}} \leq \sqrt{5 \sigma_{\varepsilon}^{2}}
$$

where the equality is satisfied when $n=1$.

Fig. 5 shows the distribution of D-Log's localization error, $\varepsilon_{\hat{d}_{t}}$, and the trend of $\sigma_{\hat{d}_{t}}$, with various $n$ values in our real-world indoor experiment environment, which is detailed in Section 4. Specifically, where $n=1$, $\sigma_{\hat{d}_{t}}$ meets the worst case with the value of 11.9 , as there is only 1 row of RSSI logs available. However, when more logs are available as shown in Fig. $5 \mathrm{~b}, \sigma_{\hat{d}_{t}}$ starts to decrease as $n$ increases. It indicates that 1$)$ as $n$ increases, $\sigma_{\hat{d}_{t}}$ decreases; 2$)$ D-Log has a floor level, which is influenced by the localization environment. 
Table 2: Aggregate statistics of the WiFi log collected in a real-world large indoor retail environment

\begin{tabular}{lr}
\hline \hline Number of user devices: & 94,396 \\
& \\
Number of AP association: & 480,924 \\
Number of Visits: & 183,745 \\
Number of WiFi APs: & 35 \\
& \\
Average of AP association per AP : & 13,741 \\
\hline \hline
\end{tabular}

\section{Data}

In this section, we present the data used for the evaluation of the performance of the proposed D-Log scheme. We evaluate the performance of the D-Log scheme in two environments: a controlled environment and a real-world large indoor environment. The complexity of the two environments is different, and so is the evaluation setup. While in the simulated environment, the mobile devices used in the training and testing set of the controlled environment are identical and therefore the variability of the used WiFi is controlled, this is not the case in the real-world large indoor environment.

\subsection{Experiment Data}

Here, we describe the experiment data from the two experimental environments: the controlled environment and the real-world large indoor environment.

For the controlled environment, we set up an experimental WLAN with 4 access points in a university meeting room (dimension: $7 \mathrm{~m}$ by $5 \mathrm{~m}$ ). We have partitioned the room into $35(1 \mathrm{~m} \times 1 \mathrm{~m})$ square grids, and used 16 of them as the test locations. These test locations were located along walls and in locations not occupied by furniture. Then, we recorded the RSSI values during handover of the carried mobile device (a smartphone) from one test AP to another. These recordings supply the training RSSI logs for D-Log scheme. For testing purposes, we collected around 6000 sample RSSI records (about 360 per location) from all detected APs, which will be used to evaluate the performance of D-Log scheme and the compared state-of-the-art localization methods.

Additionally, we have conducted real-world experiments in a large inner-city shopping mall in Sydney, Australia, covered by $67 \mathrm{WiFi}$ APs across 90,000 square meters. We used three levels of the mall to conduct our experiments, in an area of around 35,000 square meters covered by 35 WiFi APs. The WiFi log were collected from September 2012 to October 2013, and were stored in an external system. It contains around half a million AP access records from around 100,000 mobile devices. Specifically, the log includes the WiFi access point associated with the user's mobile device sampled at every 5 minutes, and the respective RSSI value for each association. These data are used as training data for the D-Log scheme with some preprocessing that is detailed in Section 4.2. Table 2 shows the statistics of the log. Note, all user identifiable information (registration details and WiFi MAC addresses) were replaced by a hash key in a non-reversible way. To examine the localization performance in this real industry environment, we selected 43 test locations across the three floors of the mall, and collected around 4000 sample RSSI records (around 100 per location) from all detected APs. Fig. 6 shows the floor maps and the test locations. Specifically, we collected 10 test locations on the $1^{\text {st }}$ floor, 15 on the $2^{\text {nd }}$ floor, and 18 on the $3^{\text {rd }}$ floor. Moreover, note this real-world RSSI log contains much complexities, which may influence all RSSI based localization methods, e.g. the variance mobile devices/antenna/Wi-Fi chipsets. There are 694 different mobile models from 53 manufacturers in our collected WiFi logs, and Table 3 and 4 show the most common manufacturers and models of the used devices in the log, respectively.

\subsection{Pre-processing the WiFi AP Log}

The real-world industry WiFi log we used was sampled at 5 minutes frequency for each user visit, and for each device, only the RSSI values for current connected AP were logged. Table 5 shows a sample of the $\log$ for a specific user. 
Table 3: Most common manufacturers of used mobile devices

\begin{tabular}{lr|lr|lr}
\hline \hline Manufacturer & $\#$ & Manufacturer & $\#$ & Manufacturer & $\#$ \\
\hline Apple & 66921 & Unidentified & 187 & Xiaomi & 22 \\
Samsung & 10587 & Huawei & 114 & Toshiba & 16 \\
Generic (Android) & 9018 & Amazon & 106 & ZTE & 13 \\
HTC & 1861 & Sony & 90 & Fujitsu & 12 \\
RIM & 1284 & Microsoft & 82 & Opera & 11 \\
SonyEricsson & 697 & Asus & 53 & KDDI & 11 \\
Nokia & 585 & Pantech & 41 & NEC & 9 \\
Google & 401 & Sharp & 35 & Alcatel & 8 \\
LG & 347 & DoCoMo & 32 & HP & 7 \\
Motorola & 240 & Acer & 26 & Lenovo & 7 \\
\hline \hline
\end{tabular}

Table 4: Most common models of used mobile devices

\begin{tabular}{|c|c|c|c|c|c|}
\hline Model & \# & Model & \# & Model & $\#$ \\
\hline iPhone (Apple) & 54873 & Galaxy Nexus (Samsung) & 420 & BlackBerry $9780(R I M)$ & 177 \\
\hline iPad (Apple) & 7523 & GT-I9305 (Samsung) & 414 & Desire HD $(H T C)$ & 173 \\
\hline iPod Touch (Apple) & 4525 & GT-I9000 (Samsung) & 407 & Desire $(H T C)$ & 159 \\
\hline Android 4.1 (Generic) & 4173 & GT-N7000 (Samsung) & 358 & PJ83100 $(H T C)$ & 145 \\
\hline GT-I9300 (Samsung) & 2791 & Fennec (Generic) & 291 & LT26i (SonyEricsson) & 142 \\
\hline GT-I9100 (Samsung) & 2602 & BlackBerry Bold Touch 9900 (RIM) & 261 & BlackBerry $9800(R I M)$ & 139 \\
\hline Android (Generic) & 1989 & Nexus 4 (Google) & 231 & Nexus S (Google) & 130 \\
\hline Android 4.0 (Generic) & 1801 & GT-S5830 (Samsung) & 220 & BlackBerry $9700(R I M)$ & 127 \\
\hline GT-N7100 (Samsung) & 849 & GT-I9305T (Samsung) & 214 & $\mathrm{~A} 510(H T C)$ & 126 \\
\hline Android 2.3 (Generic) & 452 & Unidentified (Generic) & 199 & $\mathrm{~S} 710 \mathrm{E}(H T C)$ & 124 \\
\hline
\end{tabular}

This infrequent sampling rate from single RSSI source makes it infeasible to apply existing localization methods, including trilateration, scene analysis, proximity analysis and device free method. This is because all of these existing methods require RSSI traces from multiple sources with frequent continuous sampling. So, doing localization based on this sort of data is not trivial. We conducted some data pre-precessing as follows: 1) We carry two mobile devices (one IOS iPhone 4 and one Android Sumsung S4) to the mall to record the RSSI values when a handover happens between neighbouring APs, and treat these RSSI values as the handover boundaries of corresponding APs; then 2) for each AP, we extract all the RSSI values that are less than those identified handover boundaries from the real-world WiFi log, so as to estimate the distribution of the RSSI values when handovers happen. Finally, these extracted subset of RSSI values are used as training samples for the D-Log scheme.

Table 5: Examples of the WiFi log for user E154GCHIJDESPLMX5KFJC

\begin{tabular}{cccccc}
\hline \hline Hashed MAC address & WiFi AP & RSSI & association time & disassociation time & Duration (sec) \\
\hline E154GCHIJDESPLMX5KFJC & AP 1 & -76 & $2013-02-0414: 16: 24$ & $2013-02-04 ~ 14: 21: 24$ & 300 \\
E154GCHIJDESPLMX5KFJC & AP 3 & -72 & $2013-02-0414: 21: 24$ & $2013-02-0414: 26: 24$ & 300 \\
E154GCHIJDESPLMX5KFJC & AP 7 & -75 & $2013-02-0414: 26: 24$ & $2013-02-0414: 31: 24$ & 300 \\
$\ldots$ & $\ldots$ & $\ldots$ & $\ldots$ & $\ldots$ & $\ldots$ \\
\hline \hline
\end{tabular}



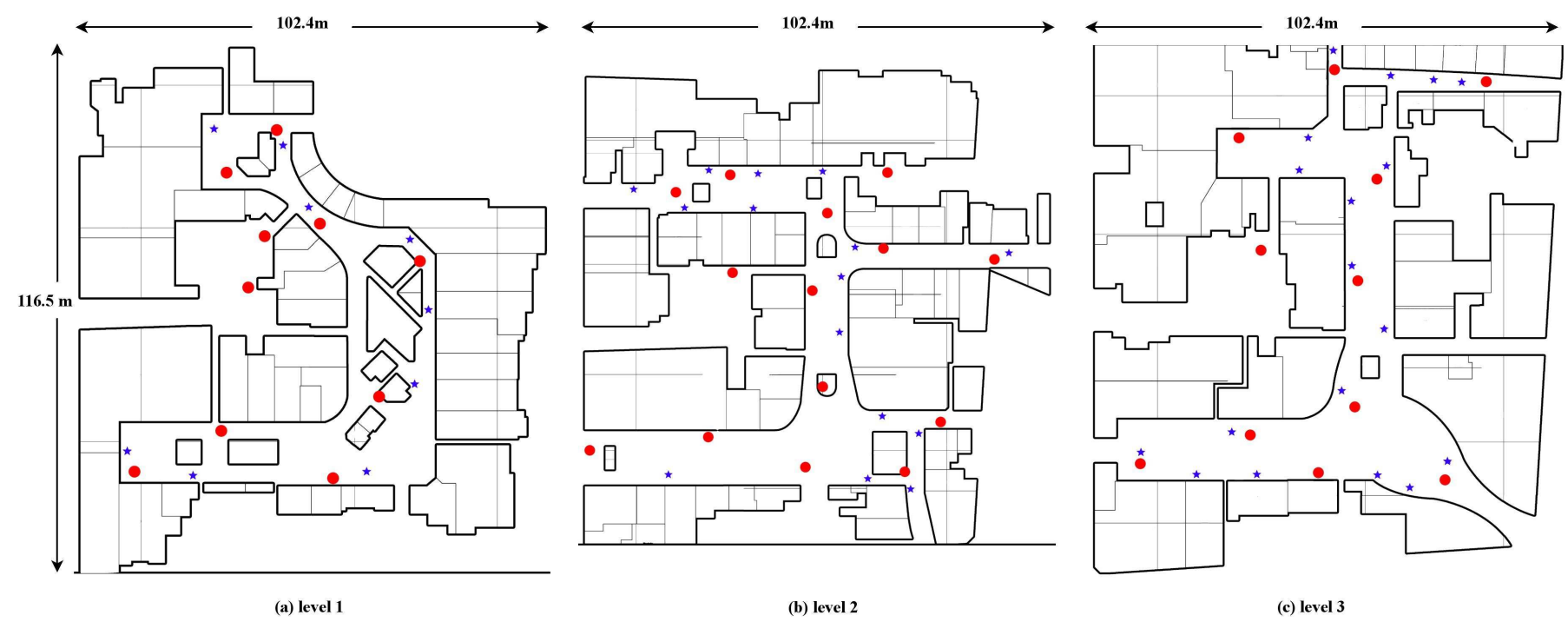

Figure 6: The floor maps in the mall where the experiments are conducted. The red dots represent the Wi-Fi APs, and the blue stars denote the test locations where ground-truth RSSI information were collected.

\section{Experiment Results}

In this section, we present the experimental configuration and the performance of the proposed DLog scheme in terms of localisation accuracy achieved by D-Log. Note, this localization relates to the determination of the distance of the mobile device from the AP and therefore the reported error indicate the width of the band in which the mobile device is located.

\subsection{Experiment Baselines}

To examine the performance of D-Log scheme thoroughly, we compare the proposed D-Log scheme with two state-of-the-art RSSI-based localization methods: scene analysis methods, and Path Loss model $[1,2]$. There are two reasons we choose these two baselines: 1) By comparing with scene analysis, we demonstrate how closely the D-Log scheme performs comparing to the state-of-the-art, because scene analysis is one of the most accurate and most popular RSSI-based localization methods; 2) the path loss model is selected to perform a fair comparison because it also makes an estimate of the radius of the receiver like the DLog scheme. For the scene analysis methods, we choose two algorithms: SVM-based method [40] and the Bayesian Network-based method [30], given that these two are among the state-of-the-art learning techniques applied for fingerprint-based indoor localization.

\subsection{Experimental Configuration}

\subsubsection{Evaluation Metrics}

The experiments were conducted on a PC running the Windows 7 Operating System with 8 GB RAM and Intel Core i7 CPU, and we conducted a 10-fold cross validation and report the results. Note that We deployed the well-known LibSVM ${ }^{1}$ package to perform the SVM-based method, and Weka (Data mining Software in Java ${ }^{2}$ ) to perform the Bayesian Network-based method; For the proposed D-Log scheme and the state-of-the-art Path Loss model, we implemented them in Java.

\footnotetext{
${ }^{1}$ https://www.csie.ntu.edu.tw/ cjlin/libsvm
}

${ }^{2}$ http://www.cs.waikato.ac.nz/ml/weka/ 
Following literature [31], we apply the mean precision $P(\mathcal{T})$ and the mean absolute error $(\varepsilon$, localization accuracy) as the measurement metric:

$$
\begin{array}{r}
P(\mathcal{T})=\frac{\left|\mathcal{T}_{c}\right|}{|\mathcal{T}|}, \\
\varepsilon=\frac{\sum\left|d_{t}-\hat{d}_{t}\right|}{|\mathcal{T}|},
\end{array}
$$

where $\mathcal{T}$ is the test set, $\left|\mathcal{T}_{c}\right|$ denotes the number of test locations that are correctly assigned to its true location, $|\mathcal{T}|$ denotes the size of $\mathcal{T}$, including both correctly assigned and incorrectly assigned test locations, $d_{t}$ is the true distance, and the $\hat{d}_{t}$ is the estimated distance. For D-Log and Path Loss model, while calculating $\left|\mathcal{T}_{c}\right|$, if $d_{t}-\sigma_{\varepsilon}<\hat{d}_{t}<d_{t}+\sigma_{\varepsilon}, \hat{d}_{t}$ is considered as the true location, otherwise false location. For SVM-based method [40] and the Bayesian Network-based method [30], they output the labels of each test location. While calculating $\varepsilon$, if the output label is the real label of the test location, $\varepsilon$ for this test location is 0 , otherwise the difference between the true distance $d_{t}$ and the distance from the AP to the output label location, which is $\hat{d}_{t}$.

\subsubsection{Parameter Estimation}

Like other localization methods, there are parameters in the proposed D-Log scheme, which are the parameters in the path loss model as shown in Eq. 1. Some of these parameters are known (e.g. the transmitter output power), or can be measured by site surveying process (e.g. path loss exponent e), but some others are hard to measure or measure accurately in practice. For example, in the investigated mall, a large variety of different brands and models of receivers (mobile phones) are involved, which makes it infeasible to measure the receiver-side related parameters; the presence of obstructions and people movement is changing frequently, which makes it hard to accurately measure other parameters, e.g. the path loss exponent $e$ and the standard deviation of shadow fading $s[2]$.

Thus, similar to other localization methods again, some data mining techniques can be applied to estimate these parameters. For example, Durgin et. al. applied linear regression to estimate the path loss exponent $e$ and the reference path loss $P L$ at $1 m$ transmitter-receiver separation by using pairwise RSSI measurements and log distances [1]. Recently, cross validation has been widely used to estimate parameters of indoor localization models, e.g. kernel-based indoor localization algorithms [41], machine learning based algorithms [42], and powerline positioning algorithms [43]. Following this, we deploy cross validation to estimate the parameters of D-Log scheme by using pairwise RSSI measurements and log distances.

Specifically, because we used the collected experimental data to both estimate the parameters of the models and evaluate them, we deployed a nested cross validation to ensure the final model evaluation is unbiased [44]. Note that, there are two disjoint datasets in D-Log scheme, the RSSI logs, and the pairwise RSSI records and distances collected at test locations. We call the RSSI logs the training set, and divide the pairwise RSSI records and distances collected at test locations into another two disjoint subsets: the validation set and the test set. Therefore, the training set, the validation set and the test set are independent to each other. Consequently, the learnt parameters will not overfit the data, and the final localization results are unbiased [44, 45].

Although theoretically the nested cross validation strategy can search and estimate the parameters in anyway, it is practically helpful to obtain the ranges of these parameters as accurate as possible. To estimate the ranges of these parameters accurately and to not disturb the investigated mall's daily business (running 7 days), we set up a shopping mall like simulation environment in the RMIT Indoor Positioning Lab. Specifically, we set up a Wi-Fi network in the simulation environment with the same configurations of that in the investigation mall, e.g. the wireless networking standard $802.11 \mathrm{n}(2.4 \mathrm{GHz})$ and the model of access points; and we used three different phones (one IOS iPhone 4, one Android Sumsung S4 and one HTC ONE) with a Java program installed to measure the receiver-side related parameters. Then, an expert, one author of this paper, measured the ranges of all parameters, which are used to determine the possible candidate values for each parameter. The detailed procedure of the deployed nested cross validation strategy is shown 
Table 6: Comparison of localization precision in controlled environment. Note, weighted D-Log, D-Log and path loss model used logs of single-AP traces; SVM-based method and Bayesian Network-Based Method used the RSSI records from multiple APs.

\begin{tabular}{c|ccccc}
\hline \hline & Weighted D-Log & D-Log & SVM-Based & Bayesian Network-Based & Path Loss \\
\hline$P(\mathcal{T})$ & $61.3 \%$ & $60.1 \%$ & $69.1 \%$ & $66.9 \%$ & $32.9 \%$ \\
\hline$\varepsilon(\mathrm{m})$ & 0.93 & 1.01 & 0.91 & 1.03 & 1.82 \\
\hline \hline
\end{tabular}

in Algorithm 1.

1 randomly divide the pairwise RSSI records and distances collected at test locations into $k$ equal sized subsets;

2 for each subset do // outer loop

3 use this subset as test set, and the rest $k-1$ subsets as validation set;

for each candidate value of the parameters in the measured ranges do // inner loop use this candidate parameter to build D-Log model on the training RSSI Logs;

validate the model on the validation set and calculate localization error for each pair of RSSI records and distances; average the localization error of all pairs to get $\varepsilon_{\text {validation }}$ on the validation set; end

select parameters that minimize $\varepsilon_{\text {validation }}$;

build model with the learnt parameters, and calculate $P(\mathcal{T})$ and $\varepsilon$ on the test set;

11 end

12 average $P(\mathcal{T})$ and $\varepsilon$ on all test set as the final result;

\section{Algorithm 1: Nested cross validation}

Note that, the training set, the validation set and the test are disjoint to each other. The deployed nested cross validation includes two loops: inner loop and outer loop. The inner loop is designed to estimate the parameters, which is a loop of a variant leave-one-out cross validation in D-Log scheme due to the following two factors: 1) the training set is always the same and is always disjoint with the validation set and the test set;2) $\varepsilon_{\text {validation }}$ is obtained by repeating and averaging the calculation of localization error on each pair of RSSI records and distances in the validation set with current parameters. The outer loop is used to evaluate the performance of the model, which is a standard $k$-fold cross validation, and we set $k=10$ in this study.

\subsection{Controlled Environment}

Here, we present the experiment results in the controlled environment, including the localization accuracy and the impact of sample size.

\subsubsection{Localization Accuracy}

Table 6 shows the results of localization precision $P(\mathcal{T})$ and $\varepsilon$ in the controlled environment. It is obtained that, for $P(\mathcal{T})$, the $c h i$-squared test shows that there is no statistical significant difference (with ch $i$-squared $=0.6735, p$-value $=0.7141$ ) between D-Log, SVM-based method, and Bayesian Network-based method. This indicates that the D-Log scheme performs well in comparison to the high-cost high-complexity scene analysis methods, SVM-based method and Bayesian Network-based method. Furthermore, the D-Log scheme performs significantly better than the path loss model. More importantly, D-Log scheme achieves similar performance to SVM-based method, Bayesian Network-based method in terms of $\varepsilon$. The weighted D-Log algorithm achieves a localization error of 0.93 meters, which is only slightly higher than that of the SVM-based method (0.91 meters); at the same time, it outperforms both Bayesian Network-based method (1.03 meters) and the Path Loss model (1.82 meters). Overall, D-Log scheme achieves comparable localization accuracy to the high-cost high-complexity localization methods. 


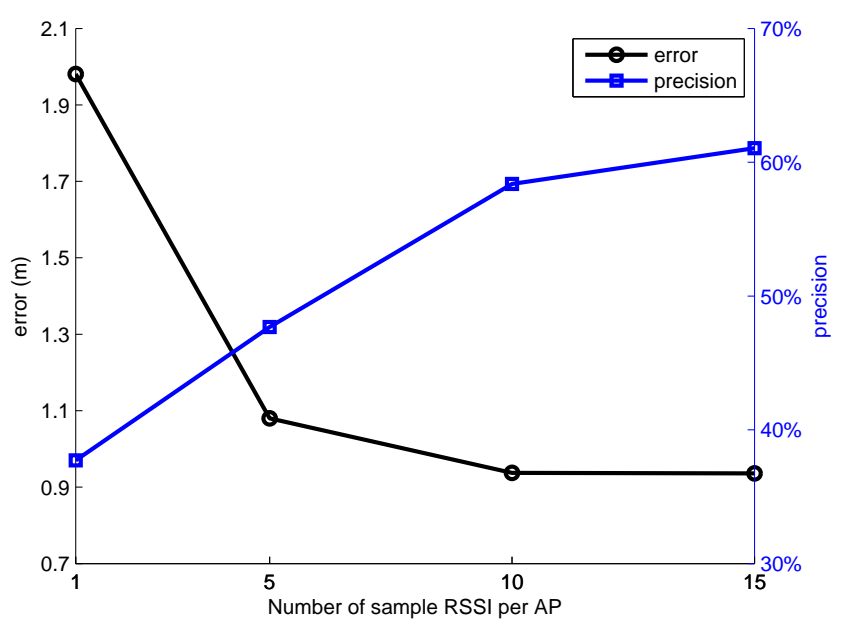

Figure 7: The impact of sample size in the controlled environment

Table 7: Single-floor localization performance in the real-world mall environment. Note, weighted D-Log, D-Log and path loss model used logs of single-AP traces; SVM-based method and Bayesian Network-Based Method used the RSSI records from multiple APs.

\begin{tabular}{cc|ccccc}
\hline \hline Floor & Metric & Weighted D-Log & D-Log & SVM-Based & Bayesian Network-based & Path Loss \\
\hline $1^{\text {st }}$ & $P(\mathcal{T})$ & $92.3 \%$ & $92.3 \%$ & $96.1 \%$ & $91.0 \%$ & $10.3 \%$ \\
& $\varepsilon(\mathrm{m})$ & 1.53 & 1.53 & 0.44 & 1.46 & 7.74 \\
\hline $2^{\text {nd }}$ & $P(\mathcal{T})$ & $81.6 \%$ & $81.6 \%$ & $89.5 \%$ & $81.6 \%$ & $21.1 \%$ \\
& $\varepsilon(\mathrm{m})$ & 2.93 & 2.93 & 1.54 & 4.09 & 8.98 \\
\hline $3^{\text {rd }}$ & $P(\mathcal{T})$ & $74.3 \%$ & $74.3 \%$ & $84.4 \%$ & $77.9 \%$ & $44.9 \%$ \\
& $\varepsilon(\mathrm{m})$ & 4.07 & 4.07 & 3.38 & 6.24 & 8.14 \\
\hline \hline
\end{tabular}

\subsubsection{Impact of Sample Size}

D-Log scheme uses the RSSI values measured during handover between two neighbouring APs, so it is important to examine the impact of the size of these sample RSSI values. Fig. 7 shows the performance of the D-Log scheme over the number of RSSI values per AP in terms of both localization precision $P(\mathcal{T})$ and error $\varepsilon$. It is observed that, as the size of training RSSI values increases, $P(\mathcal{T})$ consistently increases and $\varepsilon$ consistently decreases. This is as what we have analysed in Eq. 20 in Section 3.5, because the confidence interval of D-Log's estimation is proportional to the size of the sample observations. When only several sample observations are available, the performance is inferior, but improves and stabilizes when the sample size is greater than 10 observations in the controlled environment.

\subsection{Large Real-World Environment}

Here, we evaluate the proposed D-Log scheme in a real-world large indoor retail environment, an innercity shopping mall in Sydney, Australia, by using the anonymized real-world WiFi log of an opt-in free WiFi network operated by the mall owner. Note that this real-world mall environment is different from the environment of the department meeting room in the controlled environment, especially in terms of environment complexity, which may affect the values of RSSI readings, including brands/models of mobile devices, antenna models, Wi-Fi chipsets [46], and people movement [47] etc.

\subsubsection{Localization Accuracy}

Table 7 shows the localization accuracy in both $P(\mathcal{T})$ and $\varepsilon$ within specific single floor. Here, all compared algorithms assume the training set is restricted to the data collected on the same floor as the test location, 
Table 8: Multi-floor localization performance in the real-world mall environment. Note, weighted D-Log, D-Log and path loss model used logs of single-AP traces; SVM-based method and Bayesian Network-based method used the RSSI records from multiple APs.

\begin{tabular}{c|ccccc}
\hline \hline & Weighted D-Log & D-Log & SVM-Based & Bayesian Network-Based & Path Loss \\
\hline$P(\mathcal{T})$ & $81.1 \%$ & $81.1 \%$ & $84.3 \%$ & $82.3 \%$ & $28.4 \%$ \\
\hline$\varepsilon(\mathrm{m})$ & 3.07 & 3.07 & 2.89 & 4.3 & 8.34 \\
\hline \hline
\end{tabular}

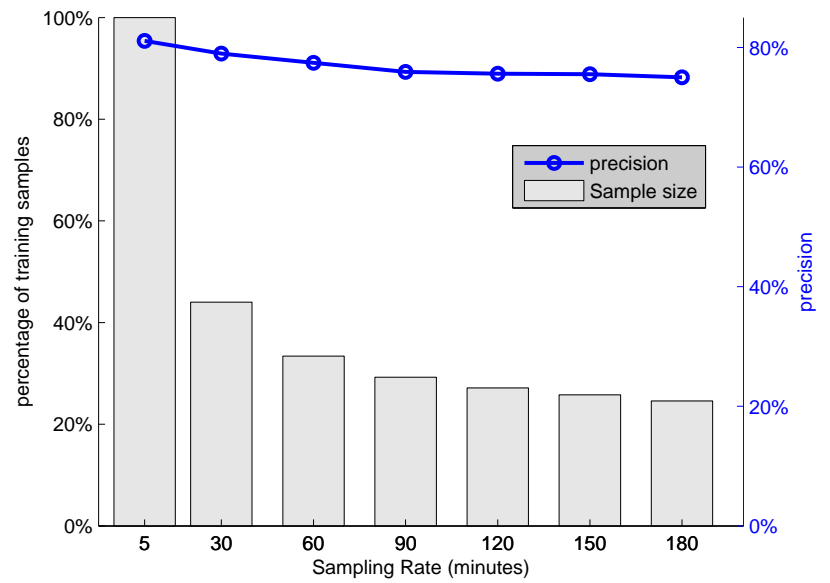

(a) Impact on $P(\mathcal{T})$

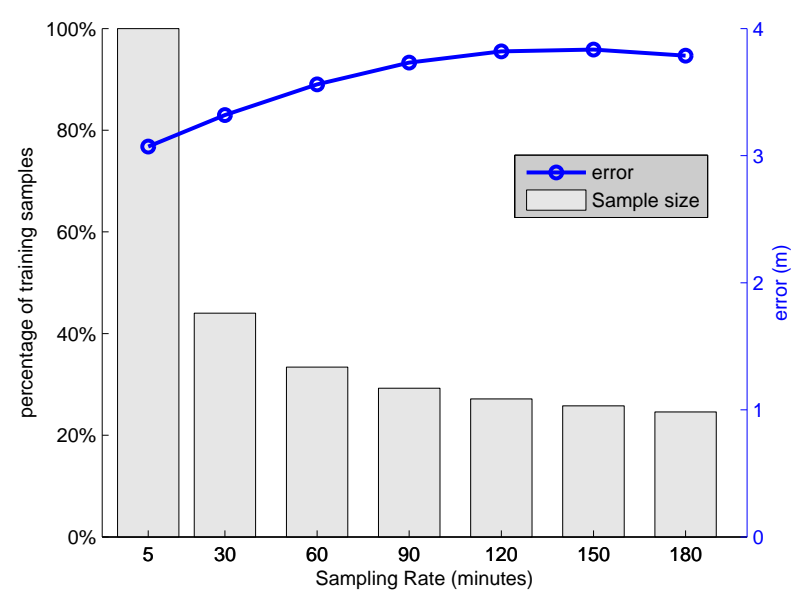

(b) Impact on $\varepsilon$

Figure 8: The impact of sampling rate in the real-world mall environment

an approaches replicated from a similar experimental environment [48]. For $P(\mathcal{T})$, it is observed that Dlog scheme performs comparatively to SVM-based method and Bayesian Network-based method across all three tested floors, and the chi-squared test results confirm that there is no significant difference in their performance: the $1^{\text {st }}$ floor $(c h i$-squared $=0.1508, p$-value $=0.9274)$, the $2^{\text {nd }}$ floor $($ chi-squared $=0.4939$, $p$-value $=0.7812)$, the $3^{r d}$ floor $(c h i$-squared $=0.6645, p$-value $=0.7173)$. For $\varepsilon$, when the complexity of the test location increases from the $1^{\text {st }}$ floor to the $3^{\text {rd }}$ floor, D-Log scheme starts outperforming the Bayesian Network-based method. This indicates that in the complex environment, some scene analysis methods will be limited to the capability of the deployed data mining method. In contrast, D-Log exhibits strong robustness in these complex environments.

Table 8 shows the results of $P(\mathcal{T})$ and $\varepsilon$ across multiple floors. To illustrate the performance of algorithms in this scenario, following [48], we remove the floor information by projecting the training points collected on different floors to a single plane, and execute all the compared algorithms. Again, the D-Log scheme significantly outperforms the Path Loss model and Bayesian network-based method, and performs comparably well to the SVM-based method.

Overall, the D-Log scheme performs comparatively to the state-of-the-art localization algorithms while utilizing less resources and being computationally less complex. In addition, we observe that in both single-floor and multiple-floor environments, weighted D-Log algorithm performs equivalently to the D-Log algorithm. This is due to the large size of the WiFi log, enabling the two methods to converge in performance.

\subsubsection{Impact of Sampling Rate}

As analysed in Section 3.5, D-Log scheme can provide accurate localization accuracy by utilizing large RSSI logs, and it is independent of the sampling rate when logging the WiFi RSSI traces. Fig. 8 shows the sample size and the $P(\mathcal{T})$ and $\varepsilon$ performance of D-Log algorithm when the sampling rate of our real-world WiFi logs varies from 5 minutes to 3 hours. The sample size is presented as the fraction of the sampling 


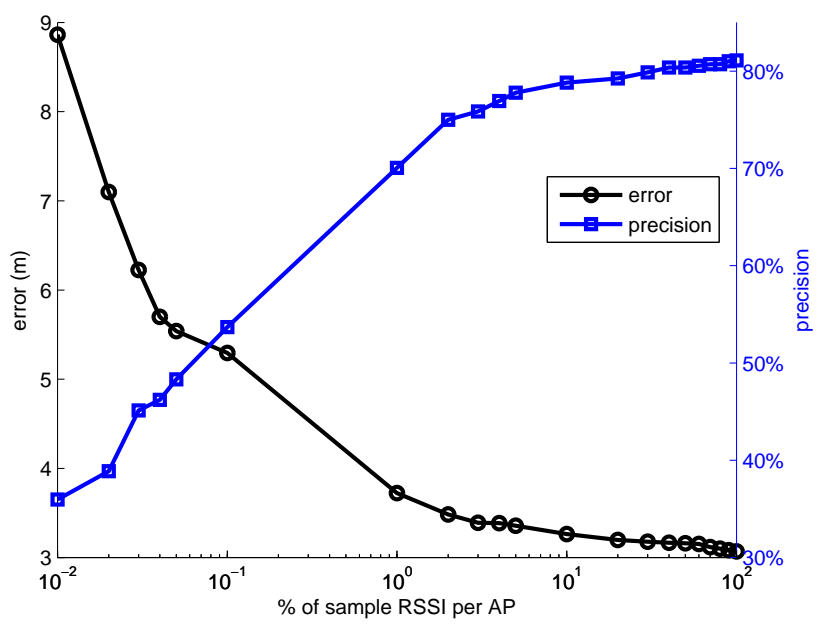

Figure 9: The impact of sample size in the real-world environment.

Table 9: Comparison of possible handover RSSI values

\begin{tabular}{c|c|ccc|c}
\hline \hline & Pre-processing & Average & Fixed $-70 d B[37]$ & $30 \%$ of least RSSIs & Path Loss \\
\hline$P(\mathcal{T})$ & $81.1 \%$ & $61.9 \%$ & $59.5 \%$ & $63.1 \%$ & $28.4 \%$ \\
\hline$\varepsilon(\mathrm{m})$ & 3.07 & 4.21 & 4.41 & 4.23 & 8.34 \\
\hline \hline
\end{tabular}

rate at the default 5 minutes. While the sampling rate drops from 5 minutes to 3 hours, $P(\mathcal{T})$ drops from $81.1 \%$ to $75.0 \%$, and $\varepsilon$ increases from 3.07 meters to 3.78 meters. In other words, while the sampling rate drops 18 times, there is no corresponding reduction in $P(\mathcal{T})$ and $\varepsilon$. This indicates that the sampling rate of the WiFi logs has little impact on the performance of D-Log scheme.

The small decrease of localization accuracy when sampling rate drops is caused by the drop of corresponding sample sizes. Specifically, when sampling rate varies from 5 minutes to 3 hours, the size of the corresponding RSSI samples drops by $75.4 \%$. A detailed discussion of the impact of sample size in this real-world environment is discussed in the following section.

\subsubsection{Impact of Sample Size in Real-World Environment}

In the real-world environment, the collected Wi-Fi logs capture heterogeneous mobile devices, thus impacting on localization. We therefore examine the impact of this noisy training sample on the performance of the D-Log scheme. Fig. 9 shows the $P(\mathcal{T})$ and $\varepsilon$ performance of D-Log in function of the training sample proportion used in the D-Log scheme, where each result in the figure is executed 10 times and then averaged. We observe that $P(\mathcal{T})$ increases proportionally with number of training samples, while $\varepsilon$ decreases, which is consistent with the findings from the controlled environment in Section 5.3. Specifically, the first several samples can largely boost the performance of the D-Log algorithm, and makes it outperform the classic path loss model; the elbow-point is achieved at around $2 \%$ of training samples, which is around 250 training samples. This indicates that in large complex environments, D-Log scheme is also robust to the noises of the training data, and can achieve accuracy comparable with competing methods with a limited number of training samples. Recall that the accuracy of the positioning relates to the determination of the distance of the mobile device from the AP, not to an exact point in $2 \mathrm{D}$ space.

\subsubsection{Impact of Handover RSSI}

To accurately estimate the distance between a mobile device and the servicing AP, D-Log scheme requires the RSSI values when handover happens between adjacent APs in the WiFi network. However, in some 


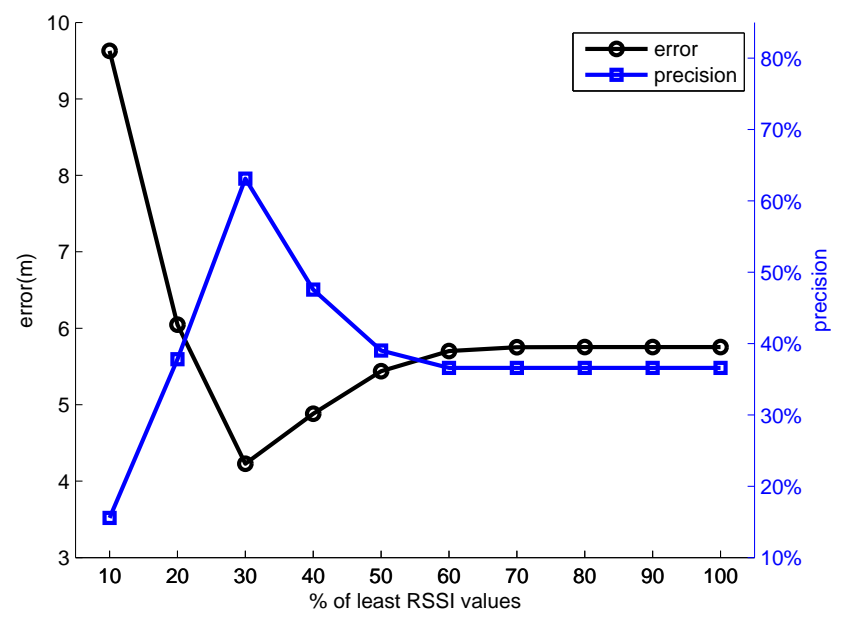

Figure 10: The impact of possible handover RSSI values

existing logs the RSSI values may be collected at very coarse frequency, e.g. the 5 minutes sampling interval in the WiFi log we experimented with. To test the applicability of such a coarsely sampled log, we have collected the accurate RSSI values at exact handover moments as a baseline (see Sec 4.2), and compared it to the subset of records estimated to have happened at, or close to, the handover. Here, we discuss the impact of the uncertainty of the handover identification on the calibration of the D-Log scheme. The baseline D-Log accuracy achieved based on the pre-processed input is compared to the following three methods:

- Average: uses the average of RSSI values of each AP in the log as the handover threshold;

- Fixed $-70 d B$ : applies a fixed value of $-70 d B$ as the handover threshold. This RSSI value is commonly suggested by commercial WiFi network installation manuals, e.g. Cisco [37];

- Least RSSI: for this method, it is assumed that the potential handovers happened when the disassociation time of $a_{x}$ is the same as the association time of $a_{y}$, which is $a_{x}$ 's adjacent AP in the WiFi network (recall, that our logs have a sampling frequency of 5mins). Then, a limited fraction of the least of these RSSI values is used to select records assumed to relate to handover RSSIs. Fig. 10 shows the performance of this method as a function of the fraction of least RSSI values. Initially, when only a small proportion (no more than 30\%) of the least RSSI values are selected, the performance increases steeply; beyond $30 \%$, the performance deteriorates.

Table 9 shows the performance of these methods in terms of $P(\mathcal{T})$ and $\varepsilon$. We observe that: 1) the D-Log scheme with the proposed pre-processing steps in Sec 4.2 achieves the best performance; 2) D-Log scheme with possible handover RSSIs, including Average, Fixed $-70 d B$ [37] and $30 \%$ of least RSSI, outperforms significantly the path loss model. This indicates that even when accurate handover RSSIs are not available, D-Log scheme still outperforms the state-of-the-art path loss model. Furthermore, with minimal environment fingerprinting that is substantially simpler than fingerprinting required by other methods, D-Log is able to achieve very good performance.

\subsection{Discussion}

The proposed D-Log scheme fulfils the five requirements introduced in the introduction of the paper:

1. non-intrusive: D-Log scheme works on the logs of discrete single-AP RSSI traces collected on the AP side, and does not need any information related with the client mobile devices, e.g. no need to install apps, or turning-on of phone sensors; 
2. generic: as long as there is an overlap between the signal coverage areas of two adjacent APs, a valid localization can be performed. Note this is generally a priority in WiFi network design. Similarly, the transmitting power of all APs is typically standard and identical for large-scale deployments and can be found in manufacturer's manuals [37];

3. light-weight: the proposed D-Log scheme is composed of simple computational components with only basic computational requirements;

4. effective: as long as a mobile device connects to the WiFi network, its RSSI value can be identified. Thus, D-Log can make a valid estimate of the radius to the connected AP;

5. accurate: the accuracy of the D-Log scheme is comparable to other state-of-the-art RSSI-based localization methods as shown by our analysis in Section 3.5, with values sufficient for applications requiring an estimate of the immediate spatial context of the user.

One limitation of the D-Log scheme is that it builds on the Path Loss model which requires certain parameters of the WiFi network to be known, as shown in Eq. 1. These parameters are known or can be measured by site surveying process, or can be learnt by using cross validation as shown in Section 5.2.2.

Due to the above discussed characteristics, D-Log can be applied in a range of applications, e.g. finegrained spatio-temporal analysis, spatial data management and indoor behaviour analysis [8]. For example, Fig. 11 shows how D-Log scheme can help when only discrete single AP-traces are available. Specifically, the figure on the left shows the D-Log's positioning of two particular mobile devices (the two purple stars). Namely, for each mobile device, the red line denotes the mean of the distribution of the estimated distance between the mobile device and its serving AP, and the pink region corresponds to the standard deviation around the estimated distance. Note that theoretically both the red line and the corresponding pink region are circular rings, but in practice this region's geometry may not resemble a circle due to some reasons, e.g. the varying signal strength distribution. The path loss model can also position the device in a similar way, but with much worse accuracy than that of D-Log, which is theoretically analysed in Section 3.5. The application of D-Log is highlighted in inset (right), showing localization improvement (the dark cyan line and the corresponding light cyan region) over simple service area positioning approximated by a Voronoi polygon [49] (thick blue line) and adjusted Voronoi regions (orange line), each centered on a single AP, that encompass all the points that are closest to that AP and accessible to the visitors based on the floorplan layout data [50]. Specifically, take the test mobile device near the bottom as an example. The corresponding adjusted Voronoi region covers around $319 \mathrm{~m}^{2}$, and D-Log positions it in a circular region of approximately $57 \mathrm{~m}^{2}$. By overlapping the D-Log positioning results with the adjusted Voronoi region, the localization of the device is improved to a more accurate region of approximately $33 \mathrm{~m}^{2}$ as shown in Fig. 11 (right). The computational cost of determining this enhanced region is only linearly proportional to the number of locations considered.

Finally, like other RSSI based localization methods, the layouts of the environment or the configurations of APs affect the proposed D-Log scheme. If they change, new AP logs need to be collected before positioning. However, the layout does not change frequently, hence data collection and model re-training will occur only as required.

\section{Conclusions}

In this paper, we investigated the following problem: How to perform accurate indoor localization using large-scale logs of discrete single-AP RSSI traces with low sampling rate? We have provided a novel means of post-hoc localization scheme, which is based on WiFi logs only, named the D-Log scheme, and proposed two algorithms: the D-Log algorithm and the weighted D-Log algorithm, with D-Log focusing on accuracy and weighted D-Log focusing on efficiency. While D-Log does not allow for the exact computation of the coordinates of the user's position, our contribution is to enhance the position estimation of post-hoc localization based on logs of single-AP traces with infrequent sampling rates. D-Log emerges as a novel means of localization enhancement which is simple and allows for improved estimation of the spatial context of the device in an indoor environment. In addition, high absolute accuracy is not always necessary. Approaches enabling contextual reasoning based on topological relationships of objects with approximate boundaries, 

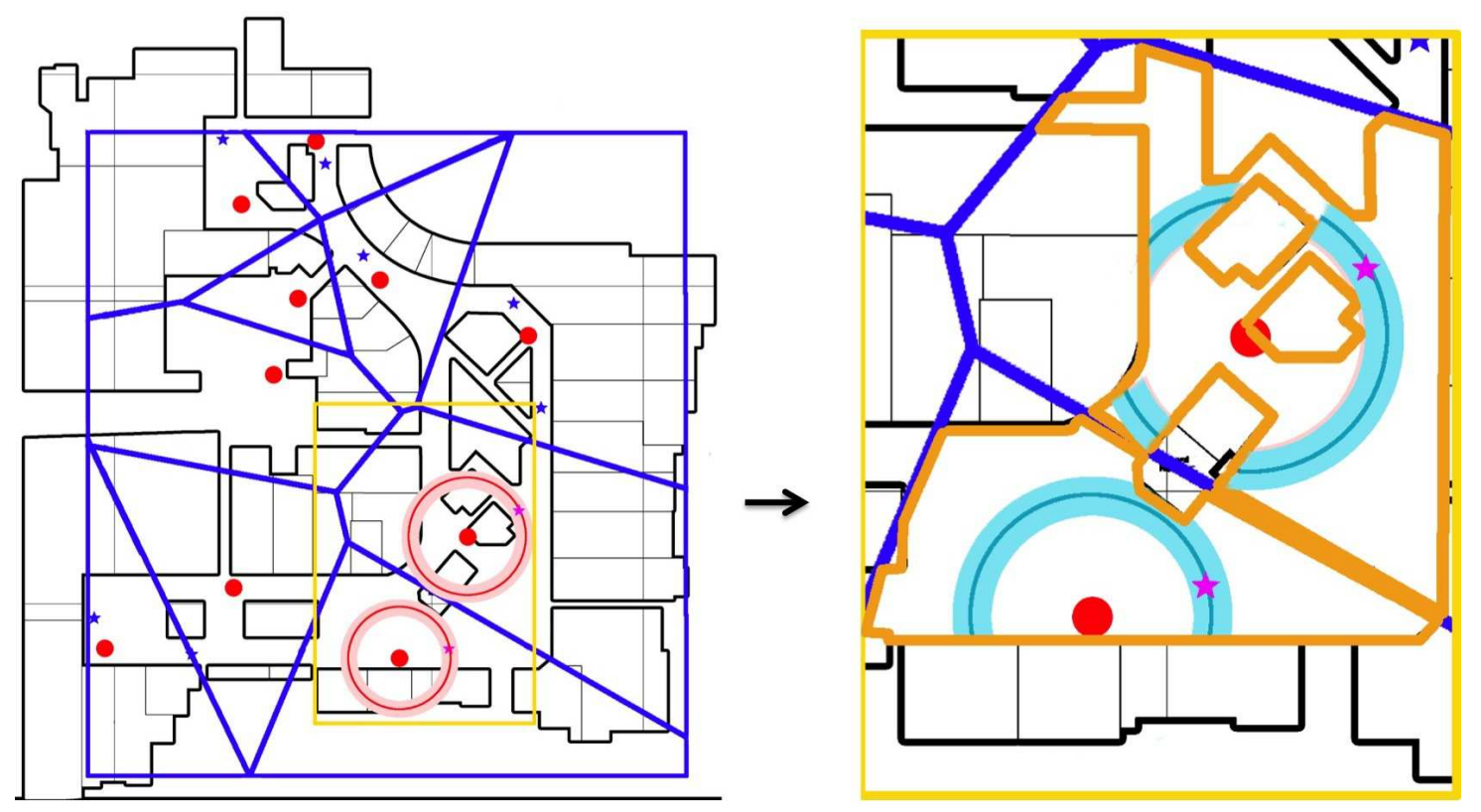

Figure 11: Illustration of the aim of D-Log (left), and how D-Log can help in reasoning about the tracked device location in spatial data management (right). The band around the ring indicates the accuracy of the D-Log positioning.

such as the egg-yolk model $[51,52]$ can be used to improve the estimate of the spatial context in which a user is active. We suggest that, by analysing spatial relations of vague regions [53], we can improve our estimates of spatial indoor behaviour of users and thus improve our estimates and predictions of indoor information needs $[54,5]$. Coupled with detailed knowledge of the environmental layout, D-Log enables a substantially improved estimation of the likely space in which a user may be located. Together with other signal about the users behaviour (movement history, web browsing logs), D-Log enables sophisticated reasoning about the users' location. Accurate estimates of the indoor context (e.g., proximity to a specific shopping mall) are critical for the improvement of indoor services and have great economical potential in the near future. In the future, we plan to combine D-Log scheme with trilateration to get better localization performance.

\section{Acknowledgement}

This research is supported by a Linkage Project grant of the Australian Research Council (LP120200413).

\section{References}

[1] G. Durgin, T. S. Rappaport, H. Xu, Measurements and models for radio path loss and penetration loss in and around homes and trees at $5.85 \mathrm{GHz}$, IEEE Transactions on Communications 46 (11) (1998) 1484-1496. doi:10.1109/26.729393.

[2] Cisco Systems Inc., Location Tracking Approaches, in: Wi-Fi Location-Based Services 4.1 Design Guide, 2008 , Ch. 2. URL http://www.cisco.com/c/en/us/td/docs/solutions/Enterprise/Mobility/WiFiLBS-DG.pdf

[3] J. A. Santana, E. Macías, Á. Suárez, D. Marrero, V. Mena, Adaptive Estimation of WiFi RSSI and Its Impact Over Advanced Wireless Services, Mobile Networks and Applications (2016) 1-13.doi:10.1007/s11036-016-0729-1.

[4] A. Suárez, J. A. Santana, E. M. Macias-Lopez, V. E. Mena, J. M. Canino, D. Marrero, RSSI Prediction in WiFi Considering Realistic Heterogeneous Restrictions, Network Protocols and Algorithms 6 (4) (2014) 19. doi:10.5296/npa.v6i4.6066.

URL http://www .macrothink.org/journal/index.php/npa/article/view/6066

[5] Y. Ren, M. Tomko, K. Ong, B. Yuntian, M. Sanderson, The influence of indoor spatial context on user information behaviours, in: M.-D. Albakour, C. Macdonald, I. Ounis, C. L. A. Clarke, V. Bicer (Eds.), Workshop on Information Access in Smart Cities, held in conjunction with the 36th European Conference on Information Retrieval ECIR 2014, ACM, 2014 
[6] A. Misra, R. K. Balan, LiveLabs: Initial reflections on building a large-scale mobile behavioral experimentation testbed, SIGMOBILE Mob. Comput. Commun. Rev. 17 (4) (2013) 47-59. doi:10.1145/2557968.2557975.

URL http://doi.acm.org/10.1145/2557968.2557975

[7] S. Scellato, M. Musolesi, C. Mascolo, V. Latora, A. T. Campbell, Nextplace: A spatio-temporal prediction framework for pervasive systems, in: Proceedings of the 9th International Conference on Pervasive Computing, Pervasive'11, SpringerVerlag, Berlin, Heidelberg, 2011, pp. 152-169.

[8] Y. Ren, M. Tomko, F. Salim, K. Ong, M. Sanderson, Analyzing Web Behavior in Indoor Retail Spaces, Journal of the Association for Information Science and Technology.

[9] C. Luo, H. Hong, M. C. Chan, PiLoc: A self-calibrating participatory indoor localization system, in: IPSN-14 Proceedings of the 13th International Symposium on Information Processing in Sensor Networks, 2014, pp. $143-153$. doi:10.1109/IPSN. 2014.6846748.

[10] K. P. Subbu, B. Gozick, R. Dantu, LocateMe: Magnetic-fields-based indoor localization using smartphones, ACM Trans. Intell. Syst. Technol. 4 (4) (2013) 73:1-73:27. doi:10.1145/2508037.2508054. URL http://doi.acm.org/10.1145/2508037.2508054

[11] A. Rai, K. K. Chintalapudi, V. N. Padmanabhan, R. Sen, Zee: Zero-effort crowdsourcing for indoor localization, in: Proceedings of the 18th Annual International Conference on Mobile Computing and Networking, Mobicom '12, ACM, New York, NY, USA, 2012, pp. 293-304. doi:10.1145/2348543.2348580. URL http://doi.acm.org/10.1145/2348543.2348580

[12] H. Wang, S. Sen, A. Elgohary, M. Farid, M. Youssef, R. R. Choudhury, No need to war-drive: Unsupervised indoor localization, in: Proceedings of the 10th International Conference on Mobile Systems, Applications, and Services, MobiSys '12, ACM, New York, NY, USA, 2012, pp. 197-210.

[13] J. T. Biehl, M. Cooper, G. Filby, S. Kratz, LoCo: A ready-to-deploy framework for efficient room localization using wi-fi, in: Proceedings of the 2014 ACM International Joint Conference on Pervasive and Ubiquitous Computing, UbiComp '14, ACM, New York, NY, USA, 2014, pp. 183-187. doi:10.1145/2632048.2636083. URL http://doi.acm.org/10.1145/2632048.2636083

[14] M. Youssef, M. Mah, A. Agrawala, Challenges: Device-free passive localization for wireless environments, in: Proceedings of the 13th Annual ACM International Conference on Mobile Computing and Networking, MobiCom '07, ACM, New York, NY, USA, 2007, pp. 222-229. doi:10.1145/1287853.1287880. URL http://doi.acm.org/10.1145/1287853.1287880

[15] A. S. Paul, E. A. Wan, F. Adenwala, E. Schafermeyer, N. Preiser, J. Kaye, P. G. Jacobs, MobileRF: A robust device-free tracking system based on a hybrid neural network HMM classifier, in: Proceedings of the 2014 ACM International Joint Conference on Pervasive and Ubiquitous Computing, UbiComp '14, ACM, New York, NY, USA, 2014, pp. 159-170. doi:10.1145/2632048.2632097. URL http://doi.acm.org/10.1145/2632048.2632097

[16] P. Castro, P. Chiu, T. Kremenek, R. R. Muntz, A probabilistic room location service for wireless networked environments, in: Proceedings of the 3rd International Conference on Ubiquitous Computing, UbiComp '01, Springer-Verlag, London, UK, UK, 2001, pp. 18-34. URL http://dl.acm.org/citation. cfm?id=647987.741335

[17] A. Ruiz-Ruiz, H. Blunck, T. Prentow, A. Stisen, M. Kjaergaard, Analysis methods for extracting knowledge from large-scale WiFi monitoring to inform building facility planning, in: 2014 IEEE International Conference on Pervasive Computing and Communications (PerCom), 2014, pp. 130-138. doi:10.1109/PerCom.2014.6813953.

[18] S. Bell, W. R. Jung, V. Krishnakumar, Wifi-based enhanced positioning systems: Accuracy through mapping, calibration, and classification, in: Proceedings of the 2Nd ACM SIGSPATIAL International Workshop on Indoor Spatial Awareness, ISA'10, ACM, New York, NY, USA, 2010, pp. 3-9.

[19] M. Azizyan, I. Constandache, R. Roy Choudhury, SurroundSense: Mobile phone localization via ambience fingerprinting, in: Proceedings of the 15th Annual International Conference on Mobile Computing and Networking, MobiCom '09, ACM, New York, NY, USA, 2009, pp. 261-272. doi:10.1145/1614320.1614350. URL http://doi.acm.org/10.1145/1614320.1614350

20] H. Bao, W.-C. Wong, A novel map-based dead-reckoning algorithm for indoor localization, Journal of Sensor and Actuator Networks 3 (1) (2014) 44-63. doi:10.3390/jsan3010044. URL http://www.mdpi.com/2224-2708/3/1/44

[21] A. T. Mariakakis, S. Sen, J. Lee, K.-H. Kim, Sail: Single access point-based indoor localization, in: Proceedings of the 12th Annual International Conference on Mobile Systems, Applications, and Services, MobiSys '14, ACM, New York, NY, USA, 2014, pp. 315-328. doi:10.1145/2594368.2594393. URL http://doi.acm.org/10.1145/2594368.2594393

[22] J. Hightower, G. Borriello, Particle filters for location estimation in ubiquitous computing: A case study, in: In Proceedings of International Conference on Ubiquitous Computing (UbiComp, 2004, pp. 88-106.

[23] I. Sabek, M. Youssef, A. Vasilakos, ACE: An Accurate and Efficient Multi-Entity Device-Free WLAN Localization System, IEEE Transactions on Mobile Computing 14 (2) (2015) 261-273. doi:10.1109/TMC.2014.2320265.

[24] A. Khan, S. K. A. Imon, S. K. Das, A novel localization and coverage framework for real-time participatory urban monitoring, Pervasive and Mobile Computing 23 (2015) 122-138. doi:10.1016/j.pmcj.2015.07.001.

[25] F. Salim, M. Williams, N. Sony, M. Dela Pena, Y. Petrov, A. A. Saad, B. Wu, Visualization of wireless sensor networks using ZigBee's received signal strength indicator (RSSI) for indoor localization and tracking, in: 2014 IEEE International Conference on Pervasive Computing and Communications Workshops (PERCOM Workshops), 2014 , pp. 575-580. doi: 10.1109/PerComW. 2014.6815270. 
[26] A. Savioli, E. Goldoni, P. Savazzi, P. Gamba, Low complexity indoor localization in wireless sensor networks by UWB and inertial data fusion, arXiv e-print 1305.1657 (may 2013).

[27] D. Hahnel, W. Burgard, D. Fox, K. Fishkin, M. Philipose, Mapping and localization with rfid technology, in: ICRA'04. 2004 IEEE International Conference on Robotics and Automation, Vol. 1, IEEE, 2004, pp. 1015-1020.

[28] Y. Zhuang, Z. Syed, J. Georgy, N. El-Sheimy, Autonomous smartphone-based wifi positioning system by using access points localization and crowdsourcing, Pervasive and Mobile Computing 18 (2015) 118-136. doi:10.1016/j.pmcj.2015.02.001.

[29] J. H, Hightower, G. Borriello, Location systems for ubiquitous computing, Computer 34 (8) (2001) 57-66.

[30] H. Liu, H. Darabi, P. Banerjee, J. Liu, Survey of wireless indoor positioning techniques and systems, IEEE Transactions on Systems, Man, and Cybernetics, Part C: Applications and Reviews 37 (6) (2007) 1067-1080. doi:10.1109/TSMCC. 2007.905750.

[31] N. Swangmuang, P. Krishnamurthy, An effective location fingerprint model for wireless indoor localization, Pervasive and Mobile Computing 4 (6) (2008) 836-850. doi:10.1016/j.pmcj.2008.04.005.

[32] E. Mok, G. Retscher, Location determination using WiFi fingerprinting versus WiFi trilateration, Journal of Location Based Services 1 (2) (2007) 145-159. doi:10.1080/17489720701781905. URL http://dx.doi.org/10.1080/17489720701781905

[33] M. Werner, L. Schauer, A. Scharf, Reliable trajectory classification using wi-fi signal strength in indoor scenarios, in: Position, Location and Navigation Symposium - PLANS 2014, 2014 IEEE/ION, 2014, pp. 663-670. doi:10.1109/PLANS.2014.6851429.

[34] A. K. M. Mahtab Hossain, H. Nguyen Van, W.-S. Soh, Utilization of user feedback in indoor positioning system, Pervasive and Mobile Computing 6 (4) (2010) 467-481. doi:10.1016/j.pmcj.2010.04.003. URL http://www.sciencedirect.com/science/article/pii/S1574119210000416

[35] B. Wang, S. Zhou, L. T. Yang, Y. Mo, Indoor positioning via subarea fingerprinting and surface fitting with received signal strength, Pervasive and Mobile Computing 23 (2015) 43-58. doi:10.1016/j.pmcj.2015.06.011.

[36] Cisco Meraki, Understanding Wireless Performance and Coverage. URL https://documentation.meraki.com/MR/WiFi _Basics_and_Best_Practices/Understanding_Wireless_Performance_and_Coverage, Tech. rep.

[37] Cisco Systems Inc., Voice over Wireless LAN 4.1 Design Guide, Tech. Rep. Cisco Validated Design I (2010).

[38] O. Chapelle, Training a support vector machine in the primal., Neural computation 19 (5) (2007) $1155-1178$. doi:10.1162/neco.2007.19.5.1155.

[39] G. K. B. Richard A. Johnsom, Statistics: Principles and Methods, 6th Edition, John Wiley and Sons, 2009.

[40] C. L. Wu, L. C. Fu, F. L. Lian, WLAN location determination in e-home via support vector classification, in: IEEE International Conference on Networking, Sensing and Control, 2004, Vol. 2, 2004, pp. 1026-1031. doi:10.1109/ICNSC. 2004.1297088.

[41] P. Agrawal, N. Patwari, Kernel Methods For RSS-Based Indoor Localization, in: S. A. R. Zekavat, R. M. Buehrer (Eds.), Handbook of Position Location: Theory, Practice, and Advances, first edit Edition, John Wiley \& Sons, Inc., 2012, Ch. 14, pp. $457-486$.

[42] H. Zou, X. Lu, H. Jiang, L. Xie, A Fast and Precise Indoor Localization Algorithm Based on an Online Sequential Extreme Learning Machine, Sensors 15 (1) (2015) 1804-1824. doi:10.3390/s150101804.

[43] S. N. Patel, K. N. Truong, G. D. Abowd, PowerLine Positioning : A Practical Sub-Room-Level, in: UbiComp'06 Proceedings of the 8th international conference on Ubiquitous Computing, 2006, pp. 441-458.

[44] B. D. Ripley, Pattern Recognition and Neural Networks, 1st Edition, Cambridge University Press, 1996.

[45] S. Arlot, A. Celisse, A survey of cross-validation procedures for model selection, Statistics Surveys 4 (2010) 40-79. arXiv:0907.4728, doi:10.1214/09-SS054 URL http://eprints.pascal-network.org/archive/00006812/

[46] G. Lui, T. Gallagher, B. Li, A. G. Dempster, C. Rizos, Differences in RSSI readings made by different Wi-Fi chipsets: A limitation of WLAN localization, in: 2011 International Conference on Localization and GNSS, ICL-GNSS 2011, 2011, pp. 53-57. doi:10.1109/ICL-GNSS.2011.5955283.

[47] J. S. C. Turner, M. F. Ramli, L. M. Kamarudin, A. Zakaria, a. Y. M. Shakaff, D. L. Ndzi, C. M. Nor, N. Hassan, S. M. Mamduh, The study of human movement effect on Signal Strength for indoor WSN deployment, in: IEEE Conference on Wireless Sensor (ICWISE), 2013, pp. 30-35. doi:10.1109/ICWISE. 2013.6728775.

[48] V. Otsason, A. Varshavsky, A. Lamarca, E. D. Lara, Accurate GSM Indoor Localization, in: Proceeding UbiComp'05 Proceedings of the 7th international conference on Ubiquitous Computing, 2005, pp. 141-158. doi:10.1007/11551201\_9.

[49] A. Okabe, B. Boots, K. Sugihara, S. N. Chiu, Spatial Tesselations: Concepts and Applications of Voronoi Diagrams, 2nd Edition, Wiley Series in Probability and Statistics, John Wiley and Sons, Ltd., Chichester, UK, 1999.

[50] Y. B. Bai, S. Wu, Y. Ren, K. Ong, G. Retscher, A. Kealy, M. Tomko, M. Sanderson, H. Wu, K. Zhang, A new approach for indoor customer tracking based on a single wi-fi connection, in: Fifth International Conference on Indoor Positioning and Indoor Navigation IPIN2014, IEEE, 2014.

[51] A. G. Cohn, N. M. Gotts, The egg-yolk representation of regions with indeterminate boundaries, Geographic objects with indeterminate boundaries 2 (1996) 171-187.

[52] T. Beaubouef, F. Petry, Vagueness in spatial data: Rough set and egg-yolk approaches, in: L. Monostori, J. Vancza, M. Ali (Eds.), Engineering of Intelligent Systems, Vol. 2070 of Lecture Notes in Computer Science, Springer, Berlin, Heidelberg, 2001, pp. 367-373. doi:10.1007/3-540-45517-5_41.

[53] A. J. Roy, J. G. Stell, Spatial relations between indeterminate regions, International Journal of Approximate Reasoning 27 (3) (2001) 205-234.

[54] Y. Ren, K. Ong, M. Tomko, M. Sanderson, How people use the web in large indoor spaces, in: 2014 ACM International Conference on Information and Knowledge Management CIKM 2014, ACM, 2014, pp. 1879-1882. 